\title{
Pullulan-coated phospholipid and Pluronic F68 complex nanoparticles for carrying IR780 and paclitaxel to treat hepatocellular carcinoma by combining photothermal therapy/photodynamic therapy and chemotherapy
}

This article was published in the following Dove Press journal:

International Journal of Nanomedicine

\begin{abstract}
Dan Wang, ',* Sipei Zhang, ,,* Tao Zhang,' Guoyun Wan,' Bowei Chen,' Qingqing Xiong, ${ }^{2}$ Jie Zhang, ${ }^{3}$ Wenxue Zhang, ${ }^{4}$ Yinsong Wang'

'Tianjin Key Laboratory on Technologies Enabling Development of Clinical Therapeutics and Diagnostics (Theranostics), School of Pharmacy, Tianjin Medical University, ${ }^{2}$ Key Laboratory of Cancer Prevention and Therapy, National Clinical Research Center for Cancer, Tianjin Medical University Cancer Institute and Hospital, ${ }^{3}$ Surgery Department, ${ }^{4}$ Radiotherapy Department, Tianjin Medical University General Hospital, Tianjin, China

*These authors contributed equally to this work
\end{abstract}

Correspondence: Yinsong Wang Tianjin Key Laboratory on Technologies Enabling Development of Clinical Therapeutics and Diagnostics (Theranostics), School of Pharmacy, Tianjin Medical University, Number 22 Qixiangtai Road, Heping District, Tianjin 300070, China

Email wangyinsong@tmu.edu.cn

Jie Zhang

Surgery Department, Tianjin Medical University General Hospital, Number 154 Anshan Street, Heping District, Tianjin 300052, China

Emailwxq_bf@I63.com

\begin{abstract}
IR780, a near-infrared dye, can also be used as a photosensitizer both for photothermal therapy (PTT) and photodynamic therapy (PDT). In this study, we designed a simple but effective nanoparticle system for carrying IR780 and paclitaxel, thus hoping to combine PTT/PDT and chemotherapy to treat hepatocellular carcinoma (HCC). This nanosystem, named PDF nanoparticles, consisted of phospholipid/Pluronic F68 complex nanocores and pullulan shells. IR780 and paclitaxel were loaded separately into PDF nanoparticles to form PDFI and PDFP nanoparticles, which had regular sphere shapes and relatively small sizes. Upon near-infrared laser irradiation at $808 \mathrm{~nm}$, PDFI nanoparticles showed strong PTT/PDT efficacy both in vitro and in vivo. In MHCC-97H cells, the combined treatment of PDFI nanoparticles/ laser irradiation and PDFP nanoparticles exhibited significant synergistic effects on inhibiting cell proliferation and inducing cell apoptosis and cell cycle arrest at G2/M phase. In MHCC97H tumor-bearing mice, PDFI nanoparticles exhibited excellent HCC-targeting and accumulating capability after intravenous injection. Furthermore, the combined treatment of PDFI nanoparticles/laser irradiation and PDFP nanoparticles also effectively inhibited the tumor growth and the tumor angiogenesis in MHCC-97H tumor-bearing mice. In summary, we put forward a therapeutic strategy for HCC treatment by combining PTT/PDT and chemotherapy.
\end{abstract}

Keywords: hepatocellular carcinoma, IR780, paclitaxel, combination therapy, nanoparticle

\section{Introduction}

Hepatocellular carcinoma (HCC) is one of the most common malignancies worldwide. In Southeast Asia and Africa, the incidence of $\mathrm{HCC}$ is expected to increase further in coming years due to increasing rate of hepatitis B and/or C virus infections. ${ }^{1,2}$ Surgery and radiofrequency ablation are highly effective for treating $\mathrm{HCC}$ in the early stage, but most patients are diagnosed at the mid or late stages of this disease. ${ }^{3,4}$ Other traditional methods such as chemotherapy and radiotherapy have not been found to be effective in prolonging the overall survival of HCC patients, meanwhile they are often associated with significant toxicities and/or acquired drug resistance. ${ }^{5,6}$ Recently, some advances have been achieved in diagnosis and therapy of HCC, but this disease still remains a serious and lethal disease due to its metastasis and recurrence.

Photothermal therapy (PTT) is a recently developed and encouraging therapeutic strategy for treatment of malignant carcinoma. This method involves photosensitizers 
to generate heat for thermal ablation under the near-infrared (NIR) laser irradiation. ${ }^{7,8}$ For cancer therapy, PTT has many advantages, for example, high specificity, minimal invasiveness and precise spatial-temporal selectivity. PTT can directly kill cancer cells in primary tumor and/or local metastasis in lymph node nearby to block the local metastasis. ${ }^{9}$ Besides that, PTT can be combined with current therapeutic modalities such as chemotherapy to combat the distant metastasis of cancer cells. Recently, the potential application of PTT in treating HCC metastasis has been explored in some groups. ${ }^{10,11}$ Some NIR small-molecule dyes have been used as PTT agents for cancer treatment. These dyes can absorb NIR laser radiation to reach an excited singlet state, and then some energy from the excited singlet state will be converted into thermal energy through vibronic relaxation or other non-radiative transitions. Moreover, the singlet state can also drop to a lower-energy-excited triplet state via intersystem crossing, thus causing the oxidation of reactive species to generate the reactive oxygen species (ROS), which can be used for the photodynamic therapy (PDT). ${ }^{12,13}$

Indocyanine green (ICG) is an only clinical NIR dye approved by the US Food and Drug Administration (FDA) for imaging and laser-mediated PTT. However, its clinical application is dramatically limited by several factors, for example, poor in vitro aqueous stability, concentrationdependent aggregation behavior, short circulation half-life, and off-target effects. ${ }^{14}$ IR780 is a recently developed NIR heptamethine dye both for PTT and PDT. Compared with ICG, IR780 is more stable and has higher florescence efficiency. Currently, IR780 has been reported to have synergistic PDT/PTT efficacy under NIR laser irradiation at $808 \mathrm{~nm} \cdot{ }^{15,16}$ Despite the promise, the clinical use of IR780 is still limited by its poor water-solubility and low tumor-targeting efficacy. Nanocarrier technologies have unique advantages for tumor-targeted delivery of drugs. First, the enhanced permeability and retention (EPR) effect of solid tumors can improve venous extravasation and tumor-accumulation of nanocarriers. Second, active tumortargeting capability of nanocarriers can be achieved by surface modification with ligands and/or antibodies for the receptors and/or epitopes of tumor tissues/or cells. Besides that, nanocarriers can enhance water-solubility of hydrophobic drugs. In view of these advantages, some nanocarriers such as nanoparticles and liposomes have been used for loading IR780, thus to improve its solubility and promote its tumor-targeted delivery, and further to increase its bioavailability, enhance its therapeutic efficacy and reduce its toxic effects. ${ }^{15-17}$
In this study, we designed a simple but effective nanoparticle system based on phospholipid, Pluronic F68 (PF68) and pullulan for carrying IR780 and chemotherapeutic drug paclitaxel (PTX), thus to realize the synergistic effects against HCC by combing PTT/PDT and chemotherapy. Neutral phospholipid 1,2-dioleoyl-sn-glycero-3-phosphoethanolamine (DOPE) and polymeric surfactant PF68 were first complexed to form DOPE/PF68 (DF) nanocores, which could greatly increase water-solubility of IR780 and PTX. Afterward, pullulan was coated outside these nanocores to form polysaccharide shells, thus obtained PDF nanoparticles. Pullulan is a natural ligand for asialoglycoprotein receptor (ASGPR) that is often overexpressed by HCC cells, ${ }^{18}$ and we have used it for HCC-targeted delivery of anticancer drugs or genes in our previous reports. ${ }^{19,20}$ Hereby, IR780- and PTX-loaded PDF nanoparticles, respectively, named as PDFI and PDFP nanoparticles, were prepared for HCC-targeted delivery and/or accumulation of these 2 drugs. Our therapeutic strategy for HCC is illustrated in Scheme 1. For eradicating HCC tumor and local metastasis, topical PTT/PDT treatment is firstly carried out by intratumoral injection of PDFI nanoparticles and followed with NIR laser irradiation at $808 \mathrm{~nm}$. For further inhibiting recurrence and distant metastasis of HCC; secondly, systemic chemotherapy is carried out by intravenous injection of PDFP nanoparticles. Through the EPR and ASGPR-mediated targeting effects, PTX can be delivered by PDFP nanoparticles to HCC tumor, which helps to exert its cytotoxic activity more efficiently. In addition, the synergistic effects of PDFI nanoparticles and PDFP nanoparticles on HCC were also investigated both in vitro and in vivo to evaluate the clinical potential of this combined treatment strategy.

\section{Materials and methods Materials}

Pullulan (average molecular weight $200 \mathrm{kDa}$ ) was provided by Hayashibara Biochemical Laboratory (Okayama, Japan). PF68 was purchased from BASF (Ludwigshafen, Germany). DOPE was obtained from Ruixi Biological Technology (Xian, China). Bovine serum albumin (BSA), 2,7-dichlorodihydrofluorescein diacetate (DCFH-DA), thiazolyl blue tetrazolium bromide (MTT), 4',6-diamidine-2'phenylindole dihydrochloride (DAPI), and IR780 iodide were purchased from Sigma-Aldrich (St Louis, MO, USA). Singlet Oxygen Sensor Green Reagent (SOSG) was purchased from Molecular Probes (Eugene, OR, USA; Thermo Fisher Scientific, Waltham, MA, USA). The LIVE/DEAD Viability/Cytotoxicity Kit was purchased from Thermo Fisher 


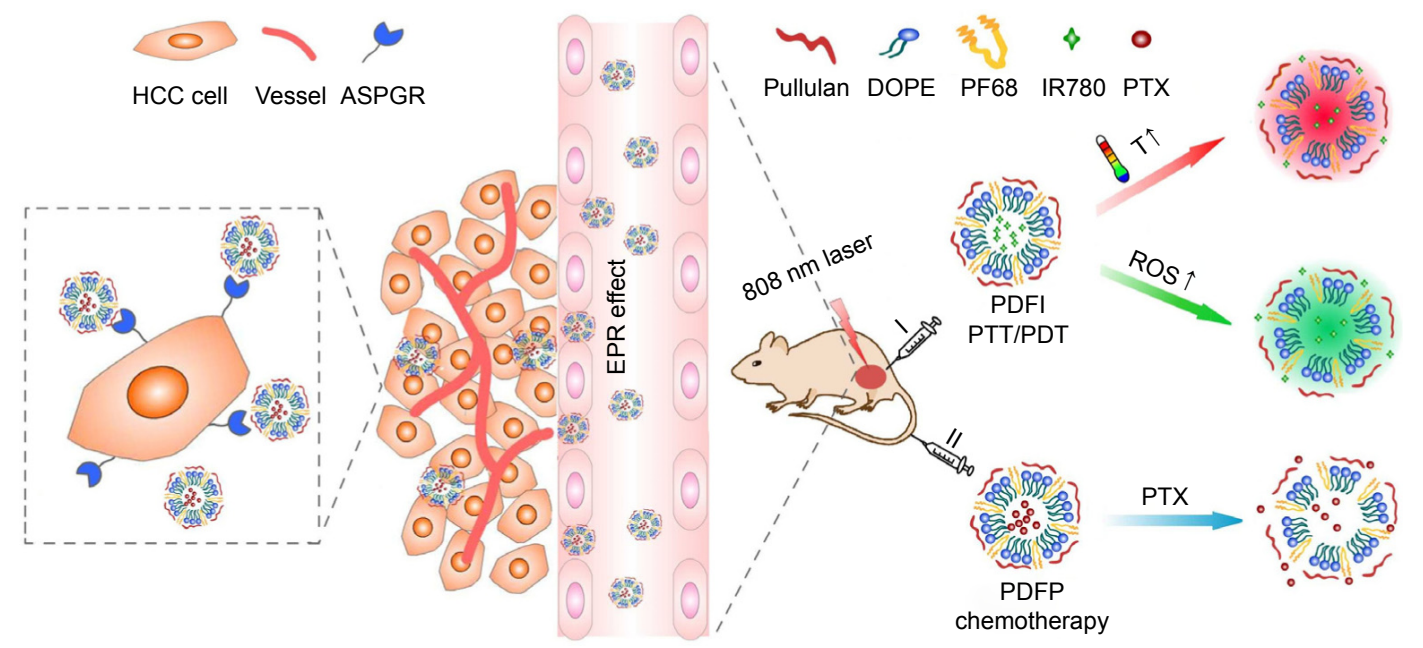

Scheme I Illustration of therapeutic strategy for HCC based on PDFI nanoparticles/NIR irradiation and PDFP nanoparticles by combining PTT/PDT and chemotherapy. Abbreviations: ASGPR, asialoglycoprotein receptor; DOPE, I,2-dioleoyl-sn-glycero-3-phosphoethanolamine; EPR, enhanced permeability and retention; HCC, hepatocellular carcinoma; I, intratumoral injection; II, intravenous injection; NIR, near infrared; PDFI, pullulan coated IR780-loaded DOPE/PF68 complex nanocore; PDFP, pullulan coated paclitaxel-loaded DOPE/PF68 complex nanocore; PF68, Pluronic F68; PDT, photodynamic therapy; PTT, photothermal therapy; PTX, paclitaxel; ROS, reactive oxygen species; $\mathrm{T}$, temperature.

Scientific Technology. All other chemical reagents were of analytical grade and obtained from the commercial sources.

Human HCC cell line MHCC-97H was obtained from American Type Culture Collection. The cells were cultured in Dulbecco's Modified Eagle's Medium (DMEM; Life Technologies, Madison, WI, USA) containing $10 \% \mathrm{v} / \mathrm{v}$ fetal bovine serum (FBS) and 1\% v/v penicillin/streptomycin. $\mathrm{BALB} / \mathrm{c}$ nude mice were bought from Vital River Laboratory Animal Technology (Beijing, China) and housed in a specific pathogen-free environment. An animal xenograft model of HCC was established by subcutaneous injection of MHCC$97 \mathrm{H}$ cells into the groin of mice at $5.0 \times 10^{6}$ cells per mouse. The animal study was approved by the Institutional Animal Care and Use Committee of Tianjin Medical University. All experimental procedures conformed to the Guide for the Care and Use of Laboratory Animals by the US National Institutes of Health (NIH Publication, 8th Edition, 2011).

\section{Methods}

\section{Preparation and characterization of blank PDF nanoparticles}

According to the previous report, ${ }^{21,22}$ a thin-film hydration method was used to prepare blank PDF nanoparticles. DOPE $5 \mathrm{mg}$ and PF68 $25 \mathrm{mg}$ were dissolved in $10 \mathrm{~mL}$ chloroform and continuously stirred for $12 \mathrm{~h}$ at room temperature. Next, the mixture was evaporated at $30^{\circ} \mathrm{C}$ under a reduced pressure to form thin films and further dried overnight in a vacuum dryer to remove the remaining solvent. After that, the dry films were hydrated in $5 \mathrm{~mL}$ deionized water in an ultrasonic cleaner at $65^{\circ} \mathrm{C}$ for $30 \mathrm{~min}$ and followed by passing through polycarbonate filter with $0.22 \mu \mathrm{m}$ pore size to obtain DF nanocores. Pullulan was then incubated with DF nanocores at different weight ratios under stirring for $4 \mathrm{~h}$, thus obtained PDF nanoparticles. All experiment steps were carried out by strictly avoiding light. The sizes, size distributions and Zeta potentials of DF nanocores and PDF nanoparticles were measured using Zetasizer Nano ZS90 apparatus (Malvern, Worcestershire, UK). Their morphologies were characterized by a transmission electron microscope (TEM, Hitachi HT7700 Tokyo, Japan). Moreover, the in vitro stability of PDF nanoparticles was evaluated by monitoring their size and zeta potential changes in $\mathrm{pH} 7.4$ phosphate buffer saline (PBS) solution during 3-day storage at $4{ }^{\circ} \mathrm{C}$.

\section{Preparation and characterization of PDFI and PDFP nanoparticles}

IR780- and PTX-loaded DF nanocores, respectively, named as DFI and DFP nanocores, were first prepared using the thin-film hydration method as described earlier. Briefly, IR780 or PTX was mixed with DOPE/PF68 in chloroform at different weight ratios and then dried to form drug-containing thin films. Afterward, these thin films were hydrated in deionized water and then passed through polycarbonate filters to form DFI or DFP nanocores. Subsequently, pullulan was incubated with these nanocores at weight ratio of 1:1, thus obtained PDFI or PDFP nanoparticles. The sizes, size distributions and zeta potentials of these nanocores and nanoparticles were determined, and meanwhile, their in-vitro stabilities were assessed in $10 \%$ FBS by detecting 
their size changes during 3-day storage at $4^{\circ} \mathrm{C}$. Furthermore, the morphologies of PDFI and PDFP nanoparticles were also characterized by TEM.

The ultraviolet-visible (UV-vis) spectroscopic method was used to determine the loading contents and encapsulation efficiencies of IR780. Briefly, DFI nanocores and PDFI nanoparticles were dissolved in dimethyl sulfoxide (DMSO) and then monitored by a UV-2450 spectrophotometer (Shimadzu, Tokyo, Japan) at $780 \mathrm{~nm}$. Moreover, the solutions free of IR780, DFI nanocores and PDFI nanoparticles were stored under the ambient light and their UV-vis absorption spectra were then recorded at 0,24 and $48 \mathrm{~h}$, thus to evaluate the photostability of these systems. Free IR780 was first dissolved in DMSO and then diluted in water containing $0.2 \%$ Tween 80 , and the volume ratio of DMSO to water was about $1 / 1$. The IR780 concentrations in all formations were $5 \mu \mathrm{g} / \mathrm{mL}$.

\section{Drug loading ability and in vitro release studies}

The loading contents and encapsulation efficiencies of PTX in DFP nanocores and PDFP nanoparticles were detected by the ultra performance liquid chromatography (UPLC) method after extraction using acetonitrile. ACQUITY UPLC system (ACQ-BSM, Waters) and C18 analytical column $(50 \times 2.1 \mathrm{~mm}, 1.7 \mu \mathrm{m}$, Waters $)$ were used and the column temperature was set at $30^{\circ} \mathrm{C}$. The mobile phase consisted of acetonitrile and deionized water $(45: 55 \mathrm{v} / \mathrm{v})$ and the flow rate was $0.2 \mathrm{~mL} / \mathrm{min}$. The injection volume was $2 \mu \mathrm{L}$ and the detection wavelength was $230 \mathrm{~nm}$. The in vitro releases of PTX from DFP nanocores and PDFP nanoparticles were evaluated in PBS solution (0.1 M, pH 7.4) containing $0.05 \%(\mathrm{w} / \mathrm{v})$ tween- 80 . The samples were transferred into dialysis bags and then dispersed in $50 \mathrm{~mL}$ release media at $37^{\circ} \mathrm{C} \pm 0.2^{\circ} \mathrm{C}$ under shaking at $100 \mathrm{rpm}$. At designated time intervals, the release media were collected through centrifugation and the fresh release media were added meanwhile. Afterward, the amounts of released PTX were determined by the UPLC method.

\section{Evaluation of in vitro and in vivo photothermal efficiencies of PDFI nanoparticles}

The in vitro PTT efficacy of PDFI nanoparticles was firstly assessed under NIR laser irradiation. Briefly, DFI nanocores and PDFI nanoparticles were diluted in PBS solution at the IR780 concentration of $100 \mu \mathrm{g} / \mathrm{mL}$ and then exposed to the NIR laser ( $808 \mathrm{~nm}, 2 \mathrm{~W} / \mathrm{cm}^{2}$ ) for $10 \mathrm{~min}$. Meanwhile, PBS solution was processed by same procedure to be used as the control. The temperature changes were recorded using a visual IR thermal imaging camera (FLIR Corporation, Wilsonville, OR, USA) every $1 \mathrm{~min}$. Next, the in vivo PTT efficacy of PDFI nanoparticles was evaluated in MHCC97H tumor-bearing mice. Briefly, $200 \mu \mathrm{L}$ solutions of DFI nanocores and PDFI nanoparticles containing $100 \mu \mathrm{g}$ of IR780 were injected into the tumors of mice directly, and meanwhile, PBS solution was used as the control. After that, the tumors were locally exposed to the NIR laser $(808 \mathrm{~nm}$, $2 \mathrm{~W} / \mathrm{cm}^{2}$ ) for $10 \mathrm{~min}$ and the temperature changes were recorded simultaneously using a visual IR thermal imaging camera every $1 \mathrm{~min}$.

\section{Evaluation of in vitro and in vivo photodynamic efficiencies of PDFI nanoparticles}

First, a fluorescence probe SOSG was used to detect the production of singlet oxygen $\left({ }^{1} \mathrm{O}_{2}\right)$ induced by PDFI nanoparticles after laser irradiation. Briefly, PBS solution (the control), DFI nanocore and PDFI nanoparticle dispersed solutions with the IR780 concentration of $100 \mu \mathrm{g} / \mathrm{mL}$ were mixed, respectively, with $25 \mu \mathrm{L}$ SOSG $(50 \mu \mathrm{M})$, and then the mixtures were exposed to the NIR laser $\left(808 \mathrm{~nm}, 2 \mathrm{~W} / \mathrm{cm}^{2}\right)$ for 2, 4, 6, 8 and $10 \mathrm{~min}$. Afterward, the produced oxidized SOSG was immediately quantified by measuring the fluorescence intensity via a microplate reader (MULTISKAN GO, Thermo Fisher Scientific, Waltham, MA, USA). The detection wavelengths were set at $504 \mathrm{~nm}$ for excitation and $525 \mathrm{~nm}$ for emission. All operations were performed strictly by avoiding light.

Second, DCFH-DA was used as a fluorescence probe to evaluate the intracellular ROS production in MHCC-97H cells after incubation with PDFI nanoparticles and followed by laser irradiation. DCFH-DA is a non-fluorescent and cellpermeant reagent. In the cells, DCFH-DA can be hydrolyzed by the esterases and its de-esterified product can further react with the intracellular ROS to produce the fluorescent compound $2^{\prime}, 7^{\prime}$-dichlorofluorescein (DCF). Briefly, the cells were seeded in the 12-well plates and treated with DFI nanocores and PDFI nanoparticles at the IR780 concentration of $2.5 \mu \mathrm{g} / \mathrm{mL}$. Some of the cells were immediately exposed to the NIR laser $\left(808 \mathrm{~nm}, 2 \mathrm{~W} / \mathrm{cm}^{2}\right)$ for $2 \mathrm{~min}$. Next, the cells with and without laser irradiation were incubated for $24 \mathrm{~h}$ and then processed with DCFH-DA reagent for $30 \mathrm{~min}$. Finally, the cells were washed with PBS solution, harvested, and analyzed by a flow cytometer (Beckman Coulter, Brea, CA, USA) to detect the intracellular fluorescence intensity of DCF.

Third, we further evaluated the in vivo PDT efficiency of PDFI nanoparticles by detecting the production of 
intratumoral ${ }^{1} \mathrm{O}_{2}$ in MHCC-97H tumor-bearing mice after administration and laser irradiation. Briefly, $200 \mu \mathrm{L}$ solutions of DFI nanocores and PDFI nanoparticles containing $100 \mu \mathrm{g}$ of IR780 were mixed with $25 \mu \mathrm{L}$ SOSG $(50 \mu \mathrm{M})$ and then injected into the tumors of mice directly. Subsequently, the tumors were locally exposed to the NIR laser $\left(808 \mathrm{~nm}, 2 \mathrm{~W} / \mathrm{cm}^{2}\right)$ for $5 \mathrm{~min}$. Next, the tumors were collected and cryosectioned onto the slides at a $5-\mu \mathrm{m}$ thickness. Afterward, the fluorescence emission of oxidized SOSG was captured by a fluorescence microscope (IX71, Olympus, Tokyo, Japan).

\section{Cellular internalization of PDFI nanoparticles}

MHCC-97H cells were seeded in the 12-well plates at a density of $5 \times 10^{3}$ cells per well and cultured overnight. After removing the culture media, the fresh culture media containing DFI nanocores and PDFI nanoparticles were added at the IR780 concentration of $2.5 \mu \mathrm{g} / \mathrm{mL}$, and the cells were further incubated for different times. Next, the cells were washed with PBS solution and then fixed with $4 \%$ paraformaldehyde for $10 \mathrm{~min}$. Finally, the cells were stained with DAPI and observed by FV-1000 confocal microscope (Olympus). The intracellular IR780 fluorescence intensities were further calculated by the software of ImageJ (National Institutes of Health, Bethesda, MD, USA).

\section{Cytotoxicity assay}

The cytotoxicities of DFI nanocores and PDFI nanoparticles in MHCC-97H cells were measured by the MTT assay. Briefly, the cells were seeded in the 96-well plates and incubated for $24 \mathrm{~h}$. After that, the culture media were replaced with the fresh culture media containing DFI nanocores and PDFI nanoparticles at different IR780 concentrations. After another 4-h incubation, some of the cells were exposed to the NIR laser ( $808 \mathrm{~nm}, 2 \mathrm{~W} / \mathrm{cm}^{2}$ ) for $2 \mathrm{~min}$ each well. Next, the cells treated with and without laser irradiation were further incubated for $24 \mathrm{~h}$ and then processed with the MTT reagent for $4 \mathrm{~h}$. Subsequently, the culture media were removed and $150 \mu \mathrm{L}$ of DMSO were added meanwhile. Finally, the absorbance of each well was measured using a ELX800 absorbance microplate reader (BioTek Epoch, Winooski, VT, USA) at $490 \mathrm{~nm}$ and the cell survival rate was then calculated. Meanwhile, the cytotoxicity of blank PDF nanoparticles was also measured by the same method.

In addition, the PTT efficacy of PDFI nanoparticles against the growth of MHCC-97H cells under the laser irradiation was assayed by the Live/Dead Cell Staining Kit. The cells were seeded into the 12 -well plate and incubated, respectively, with DFI nanocores and PDFI nanoparticles at the IR780 concentration of $2.5 \mu \mathrm{g} / \mathrm{mL}$. After incubation for $4 \mathrm{~h}$, some of the cells were exposed to a NIR laser $(808 \mathrm{~nm}$, $2 \mathrm{~W} / \mathrm{cm}^{2}$ ) for $2 \mathrm{~min}$. Next, the cells treated with and without laser irradiation were further incubated for $6 \mathrm{~h}$ and then stained with both calcein-AM and ethidium homodimer-1 for $30 \mathrm{~min}$. After washing with PBS solution, the cells were imaged using an inverted fluorescence microscope.

The synergistic effects of combined treatment of PDFI nanoparticles/laser irradiation and PDFP nanoparticles were also estimated by the MTT assay. Typically, MHCC-97H cells were seeded into the 96-well plates and incubated with PDFI nanoparticles at the IR780 concentration of $1 \mu \mathrm{g} / \mathrm{mL}$ for $4 \mathrm{~h}$. Some of the cells were exposed to the NIR laser $\left(808 \mathrm{~nm}, 2 \mathrm{~W} / \mathrm{cm}^{2}\right)$ for $2 \mathrm{~min}$, and then the cells with and without laser irradiation were treated with PDFP nanoparticles at different PTX concentrations for $48 \mathrm{~h}$. After that, the cells were processed by the same method as described previously to calculate the cell survival rates.

\section{Cell apoptosis and cell cycle analysis}

MHCC-97H cells were seeded into the 6-well plates at a density of $2 \times 10^{5}$ cells per well and cultured for $24 \mathrm{~h}$. For evaluation of PTT/PDT treatment triggered by PDFI nanoparticles on the inductions of cell apoptosis and cell cycle arrest, the cells were incubated with DFI nanocores and PDFI nanoparticles at the IR780 concentration of $1.5 \mu \mathrm{g} / \mathrm{mL}$ for $4 \mathrm{~h}$, and after that, some of the cells were exposed to the NIR laser $\left(808 \mathrm{~nm}, 2 \mathrm{~W} / \mathrm{cm}^{2}\right)$ for $2 \mathrm{~min}$ and further incubated for $24 \mathrm{~h}$. For evaluation of PDFP nanoparticles on the inductions of cell apoptosis and cell cycle arrest, the cells were incubated with free PTX and PDFI nanoparticles at the PTX concentration of $5 \mu \mathrm{g} / \mathrm{mL}$ for $48 \mathrm{~h}$. For estimating the synergistic effects of PDFI nanoparticles/laser irradiation combined with PDFP nanoparticles on cell apoptosis and cell cycle arrest, the cells were first processed with PDFI nanoparticles and 2-min laser irradiation, and then incubated with PDFP nanoparticles at PTX concentration of $5 \mu \mathrm{g} / \mathrm{mL}$ for $48 \mathrm{~h}$. Afterward, all cells were processed with Annexin V-fluorescein isothiocyanate/7amino-actinomycin $\mathrm{D}$ cell apoptosis detection kit and propidium iodide/RNase cell cycle assay kit (BD Pharmingen, San Diego, CA, USA) according to the manufacturers' instructions and finally, detected by a flow cytometer.

\section{In vivo biodistribution of PDFI nanoparticles}

The biodistribution of PDFI nanoparticles was assessed in MHCC-97H tumor-bearing mice by the in vivo imaging technique. Briefly, when the tumor volumes reached $\sim 1,000 \mathrm{~mm}^{3}$, 
the mice were injected separately with free IR780, DFI nanocores and PDFI nanoparticles via the tail vein at the IR780 dose of $0.2 \mathrm{mg} / \mathrm{kg}$. Simultaneously, the mice injected with PBS solution were used as the control. At 1, 2, 4, 8, and $24 \mathrm{~h}$ post administration, the mice were imaged using an IVIS In Vivo Imaging System (PerkinElmer, Santa Clara, CA, USA). After that, all mice were sacrificed by cervical dislocation, and then their main organs (heart, liver, spleen, lung, kidney) and tumors were collected for further observation.

\section{In vivo synergistic effects of combined treatment}

MHCC-97H tumor-bearing mice were randomly divided into 5 groups with at least 6 mice for each group and separately received treatments of normal saline (the control), free PTX, PDFP nanoparticles, PDFI nanoparticles/laser irradiation combined with and without PDFP nanoparticles. For treatments of free PTX and PDFP nanoparticles, the mice were administrated via tail vein injection at the PTX dose of $5 \mathrm{mg} / \mathrm{kg}$ every other day for consecutive 3 times. For PTT/PDT treatment, $200 \mu \mathrm{L}$ solutions of PDFI nanoparticles containing $100 \mu \mathrm{g}$ of IR780 were injected into the tumors of mice directly, and then these tumors were locally irradiated by the NIR laser $\left(808 \mathrm{~nm}, 2 \mathrm{~W} / \mathrm{cm}^{2}\right)$ for $5 \mathrm{~min}$. For combined treatment, the mice receiving PTT/PDT treatment were further treated with PDFP nanoparticles via intravenous injection at the PTX dose of $5 \mathrm{mg} / \mathrm{kg}$ for consecutive 3 times. During treatment period, the tumor sizes were monitored and the tumor volumes were calculated according to the ellipsoidal formula. ${ }^{23}$ Meanwhile, the body weights of mice were also detected. When the treatments were completed, all mice were sacrificed, and the main organs and tumors were harvested for further histological analysis.

The above tissue samples were fixed in 4\% paraformaldehyde, dehydrated with a graded ethanol series, embedded in paraffin, and cut to the sections with $5 \mu \mathrm{m}$ thickness. After that, the sections were stained with hematoxylin and eosin (H\&E; Sigma-Aldrich, St Louis, MO, USA) and then imaged with a fluorescence microscope (IX71, Olympus). The immunohistochemical staining with anti-CD31 was used to evaluate the tumor angiogenesis in the mice with various treatments. Briefly, the tumor sections were further immersed in 5\% BSA for $20 \mathrm{~min}$, and followed by incubating with rabbit polyclonal antibody against CD31 (Abcam, Cambridge, MA, USA) at 1:100 dilution at $4{ }^{\circ} \mathrm{C}$ overnight. After washing with Dako wash buffer (Dako K1492, Seoul, Korea), the sections were processed with goat anti-rabbit IgG Alexa fluor 594 red (Invitrogen A11037, Invitrogen,
Camarillo, CA, USA) at 1:500 dilution for $30 \mathrm{~min}$, dyed by diaminobenzidine and hematoxylin, and subsequently observed under a fluorescence microscope.

\section{Statistical analysis}

All values in this study were presented as mean \pm SD of at least 3 independent experiments. Statistical analysis was performed using the analysis of variance test and $p<0.05$ was considered statistically significant.

\section{Results and discussion Preparation and characterization of PDF nanoparticles}

In this study, a simple but effective nanoparticle system was developed for carrying IR780 and PTX, thus hoping to combine PTT/PDT and chemotherapy to treat HCC. This delivery system named PDF nanoparticles consisted of DF nanocores and pullulan shells. DOPE, a neutral phospholipid, is commonly used alone or with other components in the preparation of micelles, liposomes and other types of artificial membranes. ${ }^{24,25} \mathrm{PF} 68$, a triblock polymer consisting of poly(propylene oxide) block linked to 2 poly(ethylene oxide) blocks, is a nonionic polymeric surfactant and has been approved by the US FDA to be used for intravenous administration due to its high biosafety and good surface activity. Here, DOPE was complexed with PF68 to prepare DF nanocores using a thin-film hydration method. ${ }^{2,22}$ The TEM image shows that DF nanocores had a regular spherical shape with compact structure (Figure 1A). The size of DF nanocores determined by the dynamic laser scattering method was $198.3 \mathrm{~nm}$ with a relatively narrow distribution (Figure 1B). The zeta potential of DF nanocores was $+1.4 \mathrm{mV}$, indicating the electric neutrality of their surfaces.

Pullulan is a water-soluble, non-toxic, non-immunogenic, non-mutagenic and non-carcinogenic nature polysaccharide, and has become an attractive ingredient for many pharmaceutical applications and chemical manipulations. ${ }^{26}$ Pullulan has a prolonged circulation time in blood due to its electric neutrality and a specific affinity for ASGPR that is often overexpressed by hepatocyte and HCC cells. ${ }^{18,27}$ In this study, pullulan was coated on the surfaces of DF nanocores by incubation method to prepare PDF nanoparticles, thus hoping to improve HCCtargeted delivery and/or accumulation of encapsulated cargoes. The characteristic parameters of PDF nanoparticles with different weight ratios of pullulan to DF nanocores are shown in Table 1. All PDF nanoparticles had much larger sizes and negative zeta potentials compared to DF nanocores. When the weight ratio was 1/1, PDF nanoparticles displayed relatively 



D

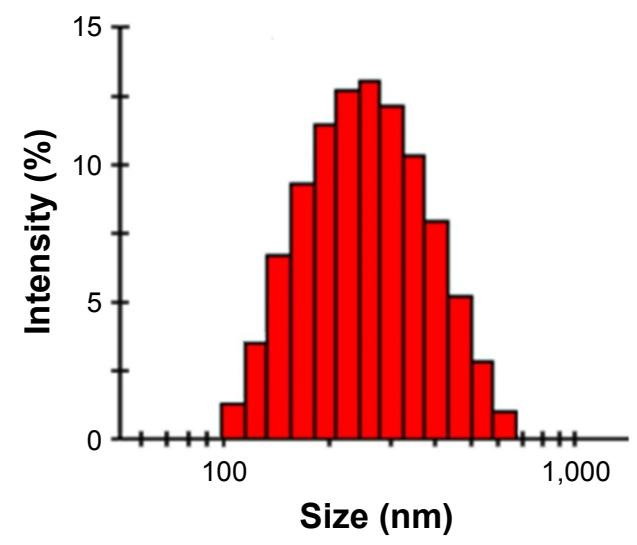

Figure I The transmission electron microscope images of DF nanocores (A) and PDF nanoparticles (B), and the size distribution histograms of DF nanocores (C) and PDF nanoparticles (D).

Abbreviations: DF, DOPE/PF68 complex; DOPE, I,2-dioleoyl-sn-glycero-3-phosphoethanolamine; PDF, pullulan coated DOPE/PF68 complex nanocore; PF68, Pluronic F68.

small size and narrow size distributions. Therefore, $1 / 1$ was believed as an optimal weight ratio of pullulan/DF nanocores for preparing PDF nanoparticles and used to carry out the following experiments. The TEM image shows that PDF nanoparticles had a clear "core-shell" structure and the thickness of pullulan shells ranged from 20 to $40 \mathrm{~nm}$ (Figure 1C).

Table I The characteristic parameters of PDF nanoparticles with different weight ratios of pullulan and DF nanocores

\begin{tabular}{llll}
\hline $\begin{array}{l}\text { Pullulan/DF } \\
\text { nanocores }(\mathbf{w} / \mathbf{w})\end{array}$ & $\begin{array}{l}\text { Size } \\
(\mathbf{n m})\end{array}$ & PDI & $\begin{array}{l}\text { Zeta potential } \\
(\mathbf{m V})\end{array}$ \\
\hline $0.25: 1$ & 200.2 & 0.538 & -2.28 \\
$0.5: 1$ & 278.5 & 0.276 & -2.08 \\
I/I & 236.8 & 0.219 & -4.17 \\
2:1 & 300.4 & 0.342 & -3.54 \\
5:I & 319.7 & 0.381 & -1.41 \\
\hline
\end{tabular}

Note: Sizes, PDIs and zeta potentials of PDF nanoparticles are presented as average values from three independent experiments.

Abbreviations: DF, DOPE/PF68 complex; DOPE, I,2-dioleoyl-sn-glycero3-phosphoethanolamine; PDF, pullulan coated DOPE/PF68 complex nanocore; PDI, polydispersity index; PF68, Pluronic F68; w/w, weight ratio.
The size of PDF nanoparticles was $248.5 \mathrm{~nm}$ with a relatively narrow distribution (Figure 1D). The zeta potential of PDF nanoparticles was $-4.17 \mathrm{mV}$. These results confirmed that pullulan was successfully coated on the surfaces of DF nanocores. Moreover, we further evaluated the in vitro stability of PDF nanoparticles by monitoring their size changes during storage. As shown in Table S1, PDF nanoparticles maintained relatively constant particle sizes in both solutions of PBS and $10 \%$ bovine serum during storage for 3 days, which suggested their good in vitro stability. In view of the aforementioned facts, we deduced that PDF nanoparticles might become a good carrier of anticancer drugs for HCC treatment.

\section{Preparation and in vitro properties of PDFI and PDFP nanoparticles}

IR780 is a heptamethine cyanine dye with the peak optical absorption at $780 \mathrm{~nm}$. Many investigations have shown that IR780 is a potential photosensitizer both for PTT and PDT 
due to its strong NIR absorption. ${ }^{15-17}$ However, IR780 has a very high hydrophobicity because of a rigid cyclohexenyl ring in its molecular structure and exhibits poor photostability in aqueous media, which will severely limit its further clinical applications. In this study, we used PDF nanoparticles to encapsulate IR780, thus improving its water solubility and enhance its photostability. Firstly, DFI nanocores were prepared by the thin-film hydration method at different feed amounts of IR780. Table S2 shows the characteristic parameters of DFI nanocores. All DFI nanocores had positive zeta potentials, which should be attributed to the positive charges in IR780 molecule. When the weight ratio of IR780/ DOPE/PF68 was 5/10/50, DFI nanocores had a small size (178.6 nm), a relatively high IR780 loading content $(8.0 \%)$ and a highest IR780 encapsulation efficiency (96.0\%). Evidently, DFI nanocores exerted the solubilization action on IR780. 5/10/50 was used as an optimal weight ratio to prepare DFI nanocores in our following experiments. PDFI nanoparticles were further prepared by incubating DFI nanocores with pullulan at the weight ratio of $1 / 1$. The loading content and encapsulation efficiency of IR780 in PDFI nanoparticles were $4.6 \%$ and $92.0 \%$, respectively (Table S3). PDFI nanoparticles had a "core-shell" spherical structure (Figure S1A), and their size and zeta potential were $248.5 \mathrm{~nm}$ and $-5.9 \mathrm{mV}$, respectively. We further evaluated the photostability of PDFI nanoparticles by testing the UV-vis spectra of IR780 after storage in light. The results are shown in Figure 2A. The maximum absorbance of free IR780 appeared at $778 \mathrm{~nm}$ and declined sharply with time. However, IR780 loaded by DFI nanocores and PDFI nanoparticles showed the absorption peak at $802 \mathrm{~nm}$, reaching a 24-nm redshift, and the decline of its absorbance slowed down significantly compared to free IR780. Especially for PDFI nanoparticles, the UV-vis spectra almost did not change during the first 24-h storage period. From the previous aforementioned results, it could be deduced that PDFI nanoparticles could effectively protect IR780 from the photolysis and the aggregation in aqueous media.
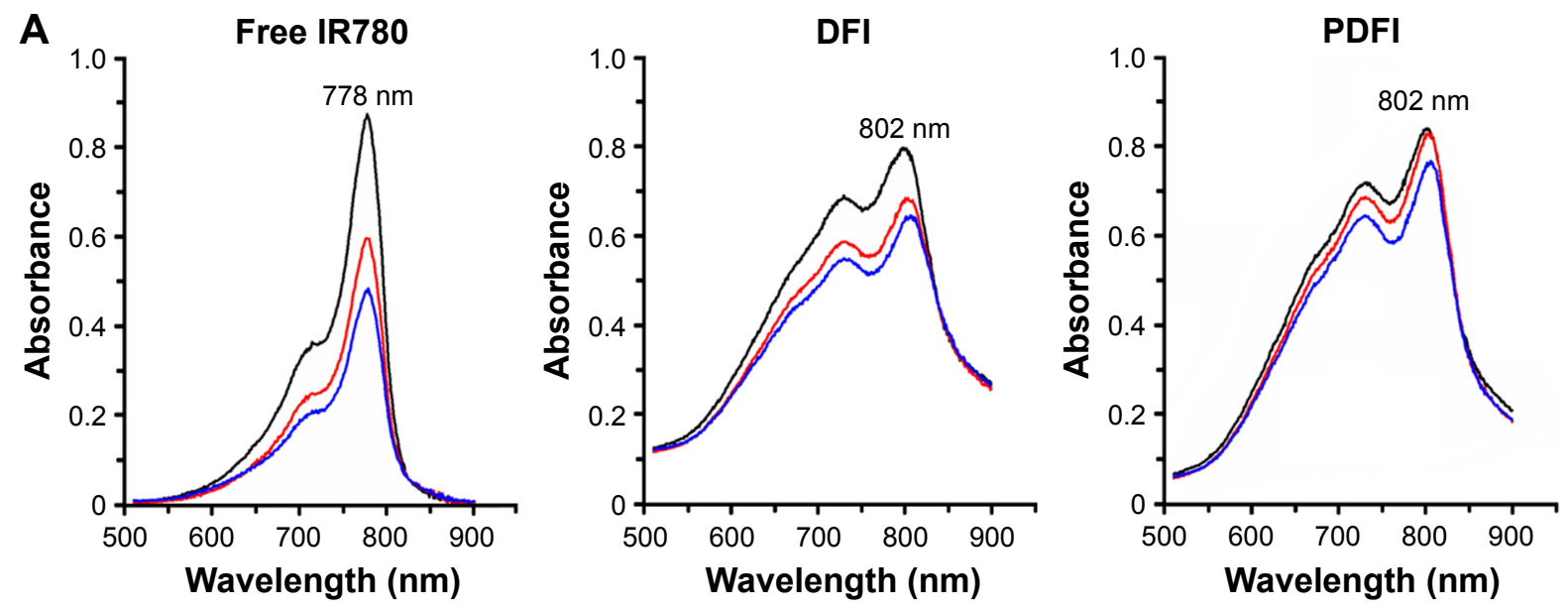

$-0 \mathrm{~h}-24 \mathrm{~h}-48 \mathrm{~h}$


Figure 2 The ultraviolet-visible spectra of free IR780, DFI nanocores and PDFI nanoparticles in water at IR780 concentration of $5 \mu \mathrm{g} / \mathrm{mL}(\mathbf{A})$, and the in vitro releases of PTX from DFP nanocores and PDFP nanoparticles in phosphate buffer saline solution at $\mathrm{pH} 7.4$ (B).

Abbreviations: DFI, IR780-loaded DOPE/PF68 complex; DFP, paclitaxel-loaded DOPE/PF68 complex; DOPE, I,2-dioleoyl-sn-glycero-3-phosphoethanolamine; PDFI, pullulan coated IR780-loaded DOPE/PF68 complex nanocore; PDFP, pullulan coated paclitaxel-loaded DOPE/PF68 complex nanocore; PF68, Pluronic F68; PTX, paclitaxel. 
For combining PTT/PDT treatment induced by PDFI nanoparticles with chemotherapy to treat $\mathrm{HCC}$, we further prepared DFP nanocores and PDFP nanoparticles. The loading contents and encapsulation efficiencies of PTX in these nanoparticles were detected by the UPLC method and the results are shown in Table S3. PDFP nanoparticles had a spherical shape and a "core-shell" structure (Figure S1B), and their size and zeta potential were $271.6 \mathrm{~nm}$ and $-6.4 \mathrm{mV}$, respectively. The electroneutral surface and the nanoscale size of PDFP nanoparticles were favor to increasing their in vivo stability and HCC-targeted delivery. As we all know, PTX is a water-insoluble drug and its solubility was only about $0.006 \mathrm{mg} / \mathrm{mL}$, thus causing its low bioavailability in vivo. ${ }^{28,29}$ However, the apparent water solubility of PTX by loading with PDFP nanoparticles was up to $1 \mathrm{mg} / \mathrm{mL}$, which would be favorable for improving the bioavailability of PTX. We further evaluated the in vitro releases of PTX from DFP nanocores and PDFP nanoparticles in $\mathrm{pH}$ 7.4 PBS and the released drug amount was monitored by the UPLC method. As shown in Figure 2B, the release profiles of PTX from DFP nanocores and PDFP nanoparticles both displayed a biphasic pattern, characterized by an initial rapid release during the first $24 \mathrm{~h}$ and followed by a more sustained release up to $120 \mathrm{~h}$. However, PTX displayed a slightly slower release from PDFP nanoparticles than that from DFP nanocores, demonstrating that PDFP nanoparticles had a higher in vitro stability due to the surface coating of pullulan.

\section{In vitro and in vivo photothermal and photodynamic efficiencies of PDFI nanoparticles}

The in vitro photothermal and photodynamic efficiencies of PDFI nanoparticles were evaluated by detecting the temperature change and the production of ${ }^{1} \mathrm{O}_{2}$ in nanoparticle systems during the 10 -min laser irradiation $\left(808 \mathrm{~nm}, 2 \mathrm{~W} / \mathrm{cm}^{2}\right)$. The IR thermal images and the temperature profiles of PBS
A
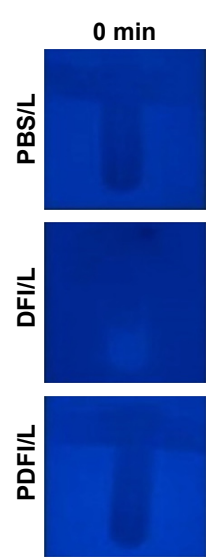

B

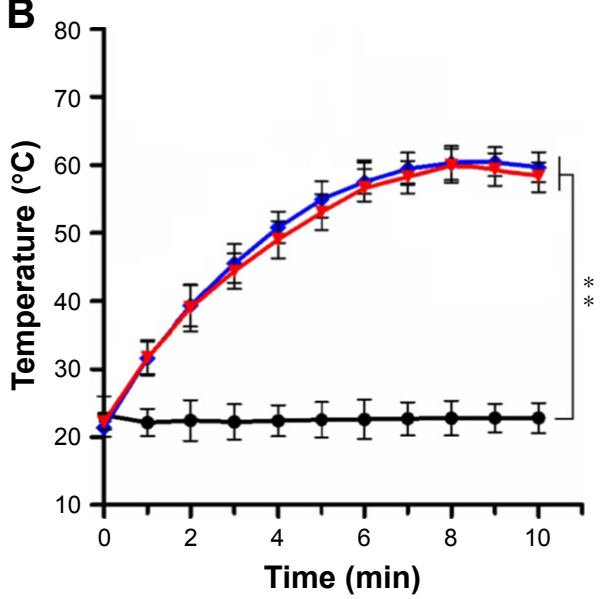

$2 \min$
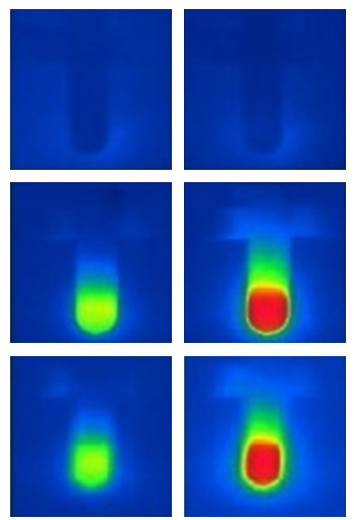

$+2$
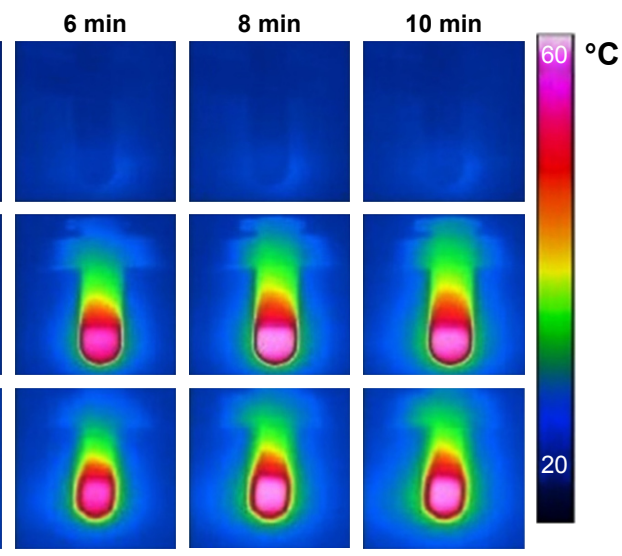

C

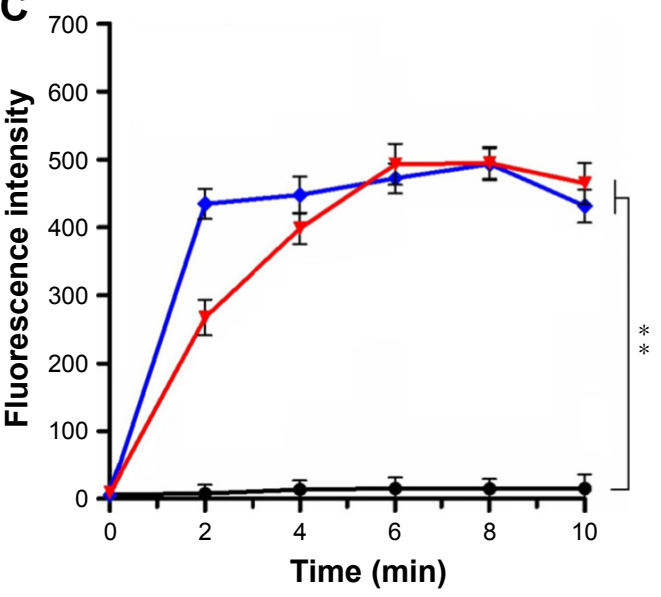

$\rightarrow \mathrm{PBS} / \mathrm{L} \rightarrow \mathrm{DFI} / \mathrm{L} \rightarrow \mathrm{PDFI} / \mathrm{L}$

Figure 3 The in vitro photothermal and photodynamic efficiencies of PDFI nanoparticles.

Notes: The infrared thermal images (A), the temperature profiles $(\mathbf{B})$ and the ' $\mathrm{O}_{2}$ generations $(\mathbf{C})$ of PBS solution, DFI nanocores and PDFI nanoparticles irradiated by an 808 $\mathrm{nm}$ laser (/L) at a powder density of $2 \mathrm{~W} / \mathrm{cm}^{2}$ for $10 \mathrm{~min}$. The concentrations of IR780 in all formulations were $100 \mu \mathrm{g} / \mathrm{mL}$. $* * p<0.0 \mathrm{I} \mathrm{compared} \mathrm{with} \mathrm{the} \mathrm{control} \mathrm{(PBS/L).}$ Abbreviations: DFI, IR780-loaded DOPE/PF68 complex; DOPE, I,2-dioleoyl-sn-glycero-3-phosphoethanolamine; PDFI, pullulan coated DFI nanocore; PF68, Pluronic F68; PBS, phosphate buffer saline. 
solution, DFI nanocores and PDFI nanoparticles are shown, respectively, in Figure $3 \mathrm{~A}$ and B. For PBS solution, a very slight temperature change was observed during the whole irradiation period. DFI nanocores and PDFI nanoparticles both showed much higher light-to-heat conversion efficiencies, for example, the temperatures of particle dispersions increased to about $60^{\circ} \mathrm{C}$ and then maintained relatively constant during the late irradiation period. It demonstrated that the photostability of IR780 was significantly enhanced by loading with DFI nanocores and PDFI nanoparticles. SOSG is a fluorescence probe for specific detection of ${ }^{1} \mathrm{O}_{2} \cdot{ }^{30,31}$ Considering that ${ }^{1} \mathrm{O}_{2}$ is a major kind of ROS species induced by PDT, we measured the ${ }^{1} \mathrm{O}_{2}$ generation in PDFI nanoparticle dispersion during the laser irradiation period using SOSG as a fluorescence probe. As shown in Figure 3C, the generations of
${ }^{1} \mathrm{O}_{2}$ in DFI nanocore and PDFI nanoparticle dispersions were significantly accelerated after the laser irradiation compared to PBS solution. Moreover, PDFI nanoparticles induced a higher level of ${ }^{1} \mathrm{O}_{2}$ generation than DFI nanocores during the early period of laser irradiation. All aforementioned results suggested that PDFI nanoparticles would be very likely to be a powerful photothermal/photodynamic agent for simultaneous PTT/PDT treatment triggered by a single NIR laser.

For further evaluating the in vivo photothermal and photodynamic efficiencies of PDFI nanoparticles, we established an animal xenograft model of HCC by subcutaneous injection of MHCC-97H cells into the groin of nude mice. The IR thermal images of mice and the temperature profiles of tumors after intratumoral injection of PBS solution, DFI nanocores and PDFI nanoparticles are shown in Figure 4A and B,
A


C
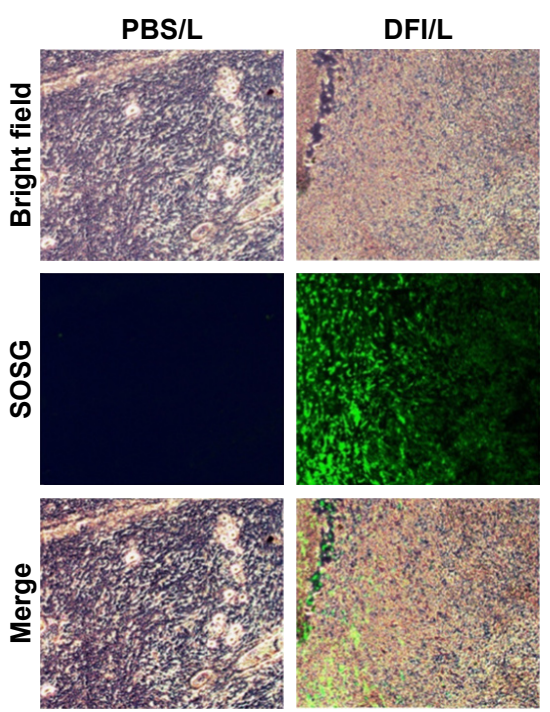

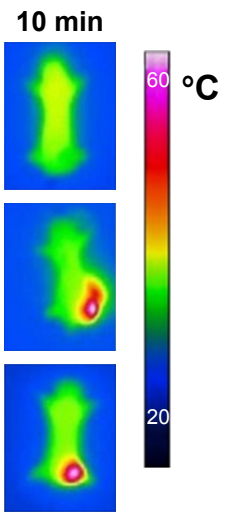

PDFI/L
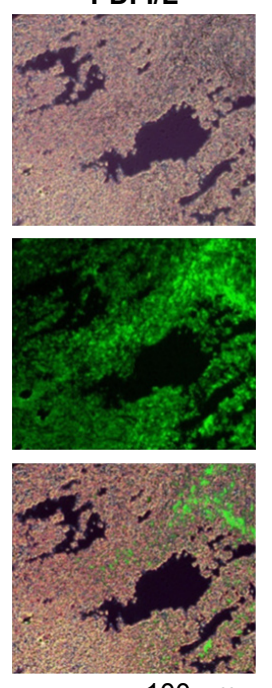

$100 \mu \mathrm{m}$
B

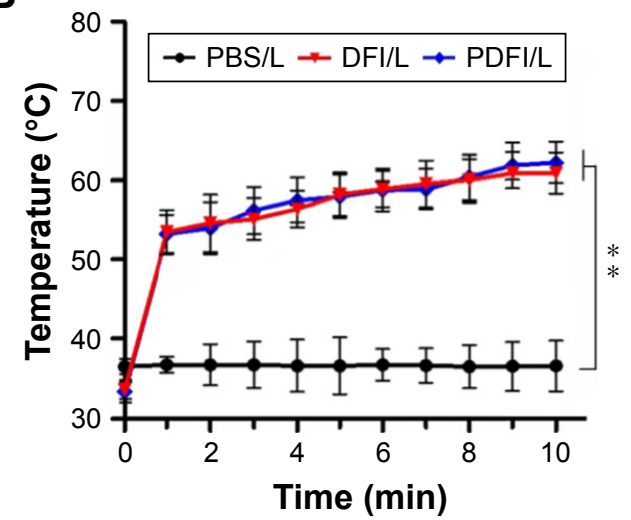

D

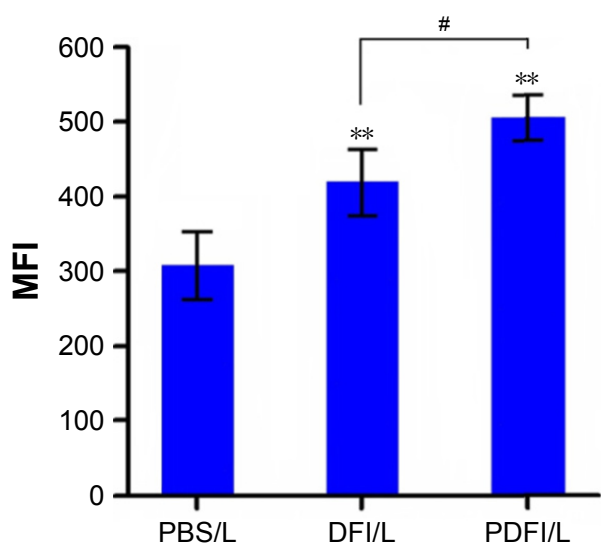

Figure 4 The in vivo photothermal and photodynamic efficiencies of PDFI nanoparticles.

Notes: (A) The infrared thermal images of mice with intratumoral injections of PBS solution, DFI nanocores and PDFI nanoparticles, and followed by the laser irradiation (/L). The temperature profiles of tumors (B), the microscopic images (C) and the comparison of fluorescence intensities (D) of SOSG-stained sections in various treatment groups. The doses of IR780 for all formulations were $100 \mu \mathrm{g}$ per mouse. The laser irradiation $\left(808 \mathrm{~nm}, 2 \mathrm{~W} / \mathrm{cm}^{2}\right)$ was carried out locally on the tumor for $10 \mathrm{~min}$. ** $<<0.0 \mathrm{I}$ compared with the control (PBS/L); ${ }^{*}<<0.05$ for comparing two treatment groups.

Abbreviations: DFI, IR780-loaded DOPE/PF68 complex; DOPE, I,2-dioleoyl-sn-glycero-3-phosphoethanolamine; MFI, mean fluorescence intensity; PF68, Pluronic F68; PBS, phosphate buffer saline; PDFI, pullulan coated DFI nanocore; SOSG, singlet oxygen sensor green reagent. 
respectively. The same results as in the aforementioned in vitro experiments were obtained. During the laser irradiation, the tumor temperatures of mice with administrations of DFI nanocores and PDFI nanoparticles constantly increased to $>60^{\circ} \mathrm{C}$, which was sufficient for tumor ablation in vivo. According to the previous report, ${ }^{32}$ SOSG can be used as a fluorescence sensor for imaging the in vivo production of ${ }^{1} \mathrm{O}_{2}$ because it could exhibit the intensive green fluorescence by oxidation with ${ }^{1} \mathrm{O}_{2}$. Thus, we used SOSG for detecting the ${ }^{1} \mathrm{O}_{2}$ generations in tumors. The images of SOSG-stained tumor sections are shown in Figure 4C. Without the laser irradiation, the tumors treated with PBS solution, DFI nanocores and PDFI nanoparticles exhibited negligible fluorescence signals (not shown). After 5-min laser irradiation $\left(2 \mathrm{~W} / \mathrm{cm}^{2}\right)$, the tumor with injection of PBS solution showed very weak fluorescence, but the tumors in all other treatment groups displayed strong green fluorescence. It indicated that these treatments triggered the generations of large amounts of ${ }^{1} \mathrm{O}_{2}$ in tumors. The comparison of fluorescence intensities is shown in Figure 4D. PDFI nanoparticles exhibited significantly higher tumor fluorescence intensity compared with DFI nanocores. We believed this was because of the enhanced tumor accumulation of PDFI nanoparticles through specific affinity for ASGPR overexpressed by HCC cells.

\section{In vitro phototherapeutic effects of PDFI nanoparticles on HCC cells}

The phototherapeutic effects of PDFI nanoparticles were evaluated in HCC MHCC-97H cells. The cell viabilities after various treatments were measured by the MTT assay and the results are shown in Figure 5A. At $24 \mathrm{~h}$ after 2-min laser irradiation $\left(808 \mathrm{~nm}, 2 \mathrm{~W} / \mathrm{cm}^{2}\right)$, DFI nanocores and PDFI nanoparticles all significantly inhibited the growths of MHCC-97H cells in an IR780 concentration-dependent manner. However, blank PDF nanoparticles either combined with or without laser irradiation did not significantly influence the cell growth in nanoparticle concentration range of $0.2-2 \mathrm{mg} / \mathrm{mL}$ (data not shown). It indicated that the photothermal and photodynamic effects of PDFI nanoparticles played important roles in suppressing the cell growth. Compared with DFI nanocores, PDFI nanoparticles displayed slightly lower cytotoxicites in both cases of combining with and without laser irradiation, suggesting that the phototoxicity of IR780 was reduced by loading with PDFI nanoparticles. To visually estimate the cytotoxicity of PDFI nanoparticles combined with laser irradiation, the cells were processed with the LIVE/DEAD Viability/Cytotoxicity Kit, in which calcein-AM and ethidium homodimer-1 were used to identify the live and dead cells, respectively. As shown in Figure 5B, strong green florescence was observed in the cells only treated with DFI nanocores and PDFI nanoparticles at the IR780 concentration of $2.5 \mu \mathrm{g} / \mathrm{mL}$, but the cells with further treatment of 2-min irradiation were partially dead and showed intensive red florescence signals.

The photothermal and photodynamic efficacies of PDFI nanoparticles were next evaluated in MHCC-97H cells by detecting the temperature changes by an IR camera and the intracellular productions of ROS using DCFH-DA as a fluorescence probe. After laser irradiation, the temperatures of cell cultures containing DFI nanocores and PDFI nanoparticles were rapidly raised from $37^{\circ} \mathrm{C}$ to $42^{\circ} \mathrm{C}$, which was one of the major reasons to cause the death of cells. DCFH-DA is a non-fluorescent and cell-permeant reagent. After entering the cells, DCFH-DA can be hydrolyzed by the esterases and its de-esterified product can be further converted into the fluorescent compound DCF upon oxidation with intracellular ROS. ${ }^{33}$ Therefore, the ROS productions in MHCC-97H cells after various treatments could be assessed by detecting the intracellular fluorescence intensities of DCF using the flow cytometry. The results are shown in Figure 5C. Compared with the control, DFI nanocores and PDFI nanoparticles all increased the intracellular ROS levels, which was perhaps due to the stress response of HCC cells. After the laser irradiation, the ROS productions in these treated cells were further remarkably enhanced, demonstrating strong photodynamic efficacies of these treatments against HCC cells. Moreover, PDFI nanoparticles exhibited a significantly higher PDT efficiency in the cells compared with DFI nanocores. We believed it was because those PDFI nanoparticles were internalized into HCC cells more efficiently through the specific affinity of their polysaccharide shells for ASGPR.

Therefore, we further evaluated the uptakes of PDFI nanoparticles in MHCC-97H cells by the confocal microscopy using the autofluorescence of IR780. Figure 5D displays the confocal images of cells incubated with DFI nanocores and PDFI nanoparticles. At $4 \mathrm{~h}$ after incubation, the yellow fluorescence from IR780 was mainly located in the cytoplasm, and PDFI nanoparticles exhibited more intensive fluorescence than DFI nanocores at the same IR780 concentrations. Figure 5E shows the comparison of intracellular fluorescence intensities after various treatments. DFI nanocores and PDFI nanoparticles showed almost identical intracellular fluorescence intensities during the first $0.5 \mathrm{~h}$. However, the intracellular fluorescence signals of PDFI nanoparticles were significantly stronger than that of DFI nanocores after $1 \mathrm{~h}$. These results confirmed our speculation 

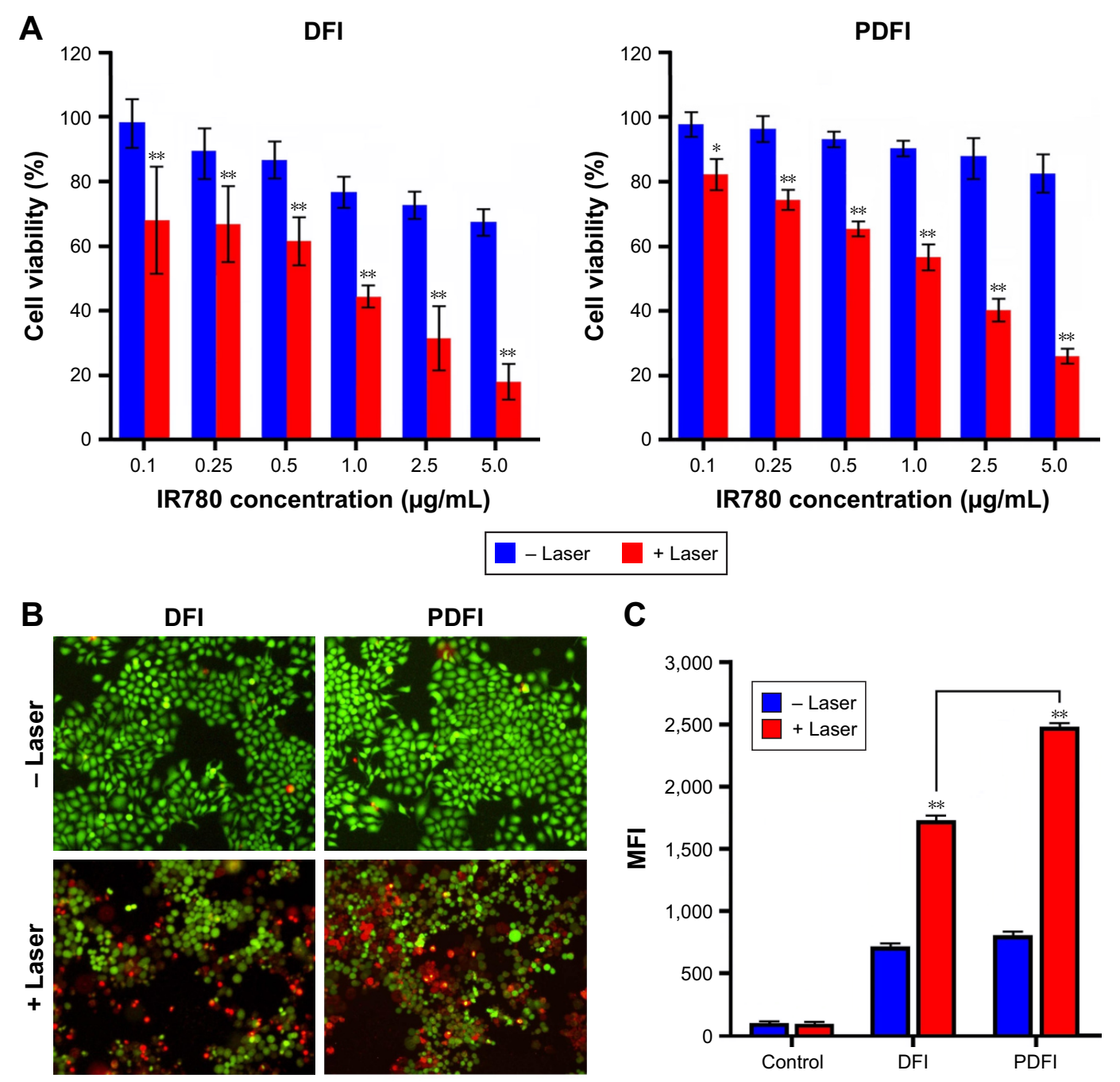

+ Laser

C

$200 \mu \mathrm{m}$

D $\quad 0.5 \mathrm{~h}$


$1 \mathrm{~h}$
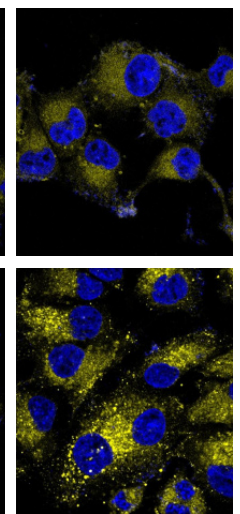

$4 \mathrm{~h}$
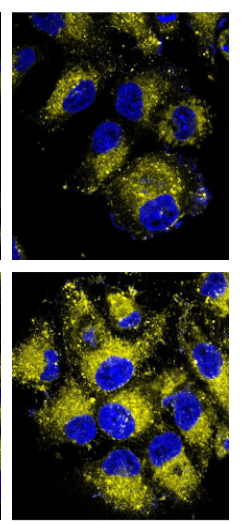

$50 \mu \mathrm{m}$

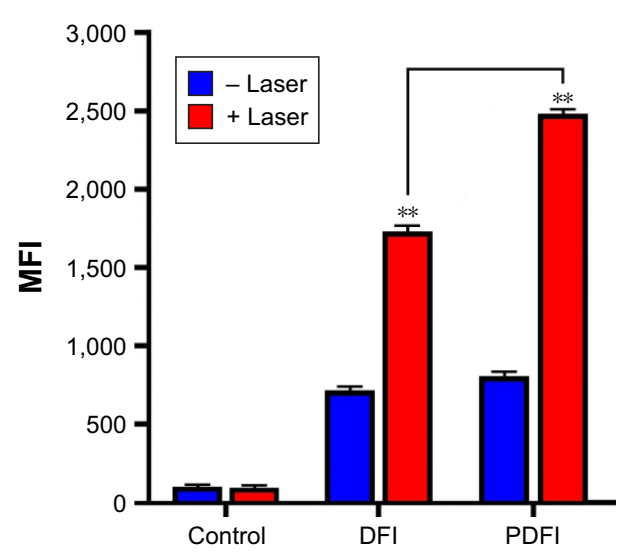

$\mathbf{E}$

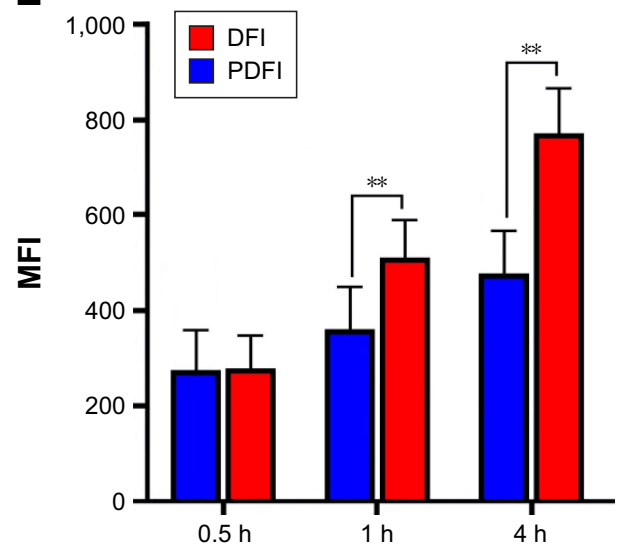

Figure $\mathbf{5}$ The cytotoxicity and uptake of PDFI nanoparticles in MHCC-97H cells.

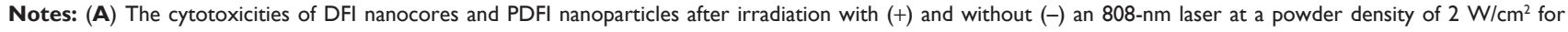
2 min. $* p<0.05$ and $* * p<0.01$ for comparing treatments with and without laser irradiation. (B) The fluorescence microscopy images of cells with various treatments. The concentration of IR780 was $2.5 \mu \mathrm{g} / \mathrm{mL}$. The live cells showing green fluorescence were stained with calcein-AM and the dead cells showing red fluorescence were stained with ethidium homodimer-I. (C) The comparison of MFIs of intracellular DCF produced from the reaction of DCFH-DA with ROS after various treatments. (D) The confocal images of cells incubated with DFI nanocores and PDFI nanoparticles for different times at the IR780 concentration of $2.5 \mu \mathrm{g} / \mathrm{mL}$. (E) The comparison of MFIs of IR780 in cells after various treatments. ** $p<0.01$ for comparing two treatment groups.

Abbreviations: DCF, 2',7'-dichlorofluorescein; DCFH-DA, 2'-7'-dichlorodihydrofluorescein diacetate; DFI, IR780-loaded DOPE/PF68 complex; DOPE, I,2-dioleoyl-snglycero-3-phosphoethanolamine; MFI, mean fluorescence intensity; PDFI, pullulan coated DFI nanocore; PF68, Pluronic F68; ROS, reactive oxygen species. 
that PDFI nanoparticles were internalized into MHCC-97H cells more efficiently for their pullulan shells, which would be favorable for HCC-targeting therapy.

\section{In vitro synergistic effects of PDFI nanoparticles/laser irradiation combined with PDFP nanoparticles}

Many investigations have shown that PTT and/or PDT combined with chemotherapy could suppress tumor recurrence and metastasis more effectively than these treatments alone. ${ }^{34-36}$ In this study, we hoped to combine photothermal/ dynamic effects of PDFI nanoparticles/laser irradiation and cytotoxic activity of PDFP nanoparticles to treat HCC. First, we evaluated the synergistic effects of combined treatment on the growth of MHCC-97H cells by the MTT assay. After incubation for $48 \mathrm{~h}$, free PTX inhibited the cell growth in a concentration-dependent manner and the $\mathrm{IC}_{50}$ was about $10.5 \mu \mathrm{g} / \mathrm{mL}$ (Figure 6A). PDFP nanoparticles exhibited slightly decreased cytotoxicity and the $\mathrm{IC}_{50}$ was higher than $12 \mu \mathrm{g} / \mathrm{mL}$ PTX (Figure 6B). However, combined with PDFI nanoparticles ( $1 \mu \mathrm{g} / \mathrm{mL}$ IR780) and followed by laser irradiation ( $2 \mathrm{~W} / \mathrm{cm}^{2}, 2 \mathrm{~min}$ ), PDFP nanoparticles had a significantly enhanced cytotoxicity and the $\mathrm{IC}_{50}$ was only about $5.0 \mu \mathrm{g} / \mathrm{mL}$ PTX (Figure 6B). It thus could be deduced that this combined treatment possessed significant synergistic effects on the growth of HCC cells.

Next, we assessed the apoptosis induction of PDFI nanoparticles/laser irradiation combined with PDFP nanoparticles
A

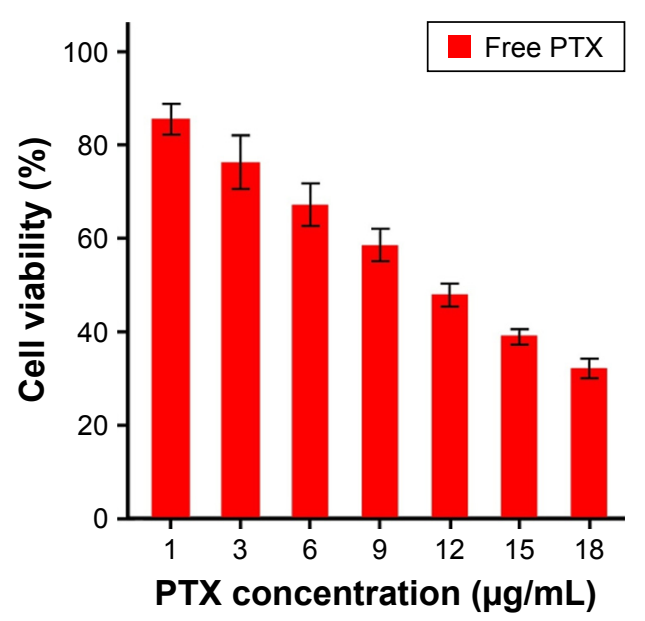

C

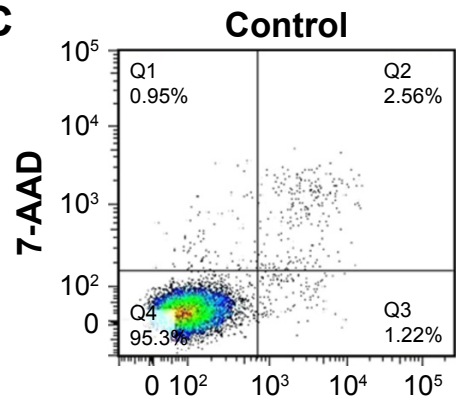

Annexin V-FITC

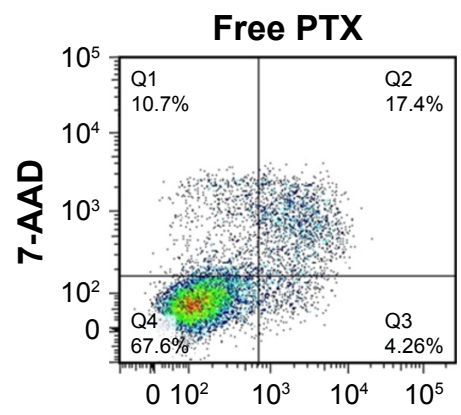

Annexin V-FITC

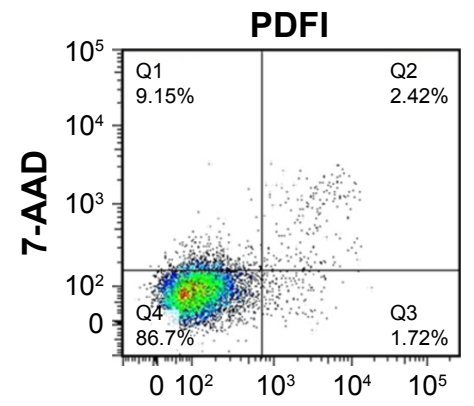

Annexin V-FITC

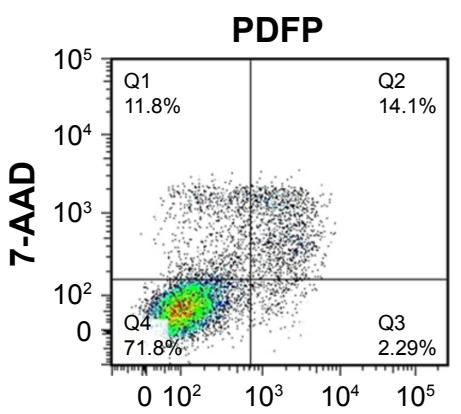

Annexin V-FITC
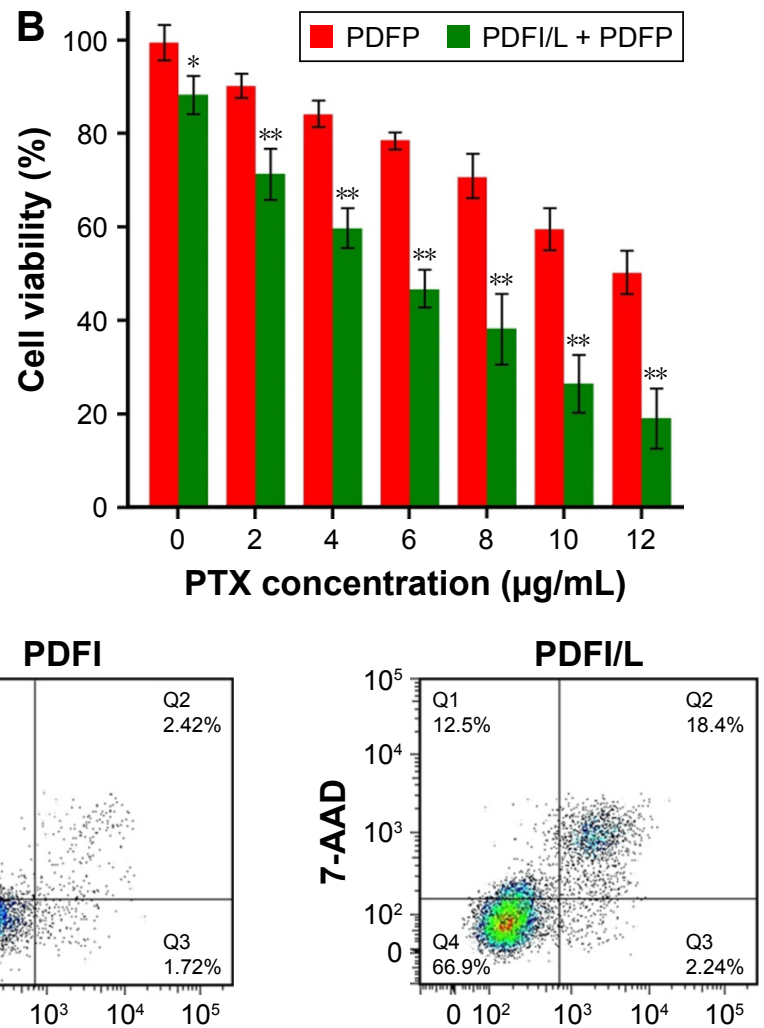

Annexin V-FITC

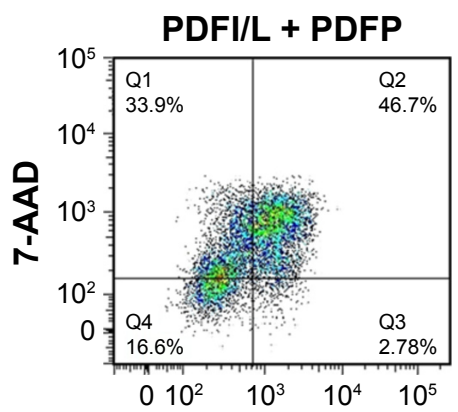

Annexin V-FITC

Figure 6 (Continued) 


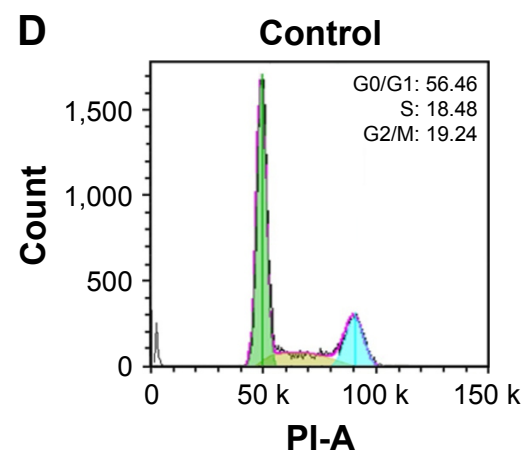

Free PTX

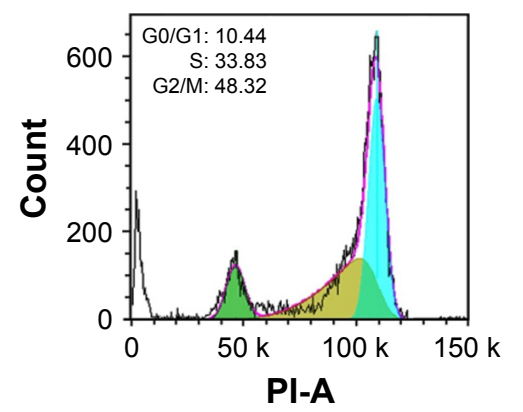

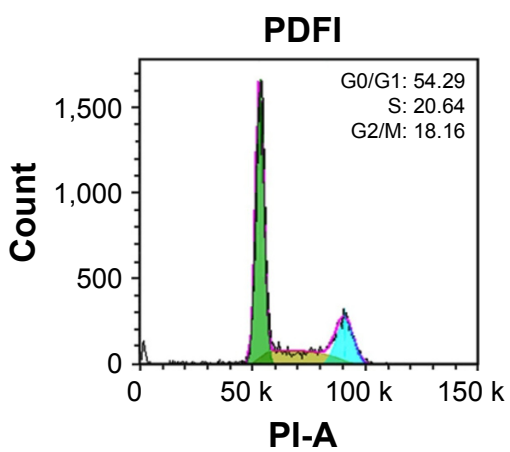

PDFP

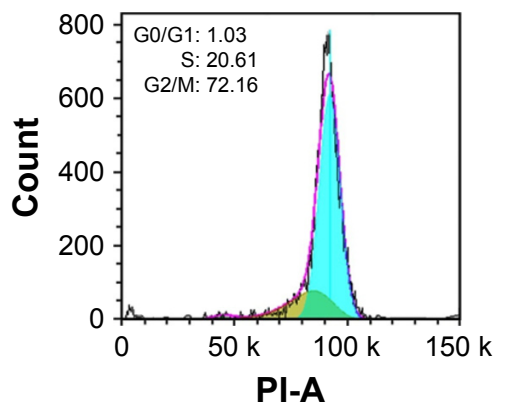

PDFI/L

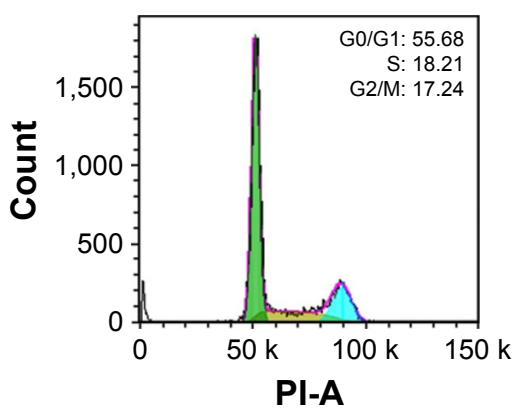

PDFI/L + PDFP

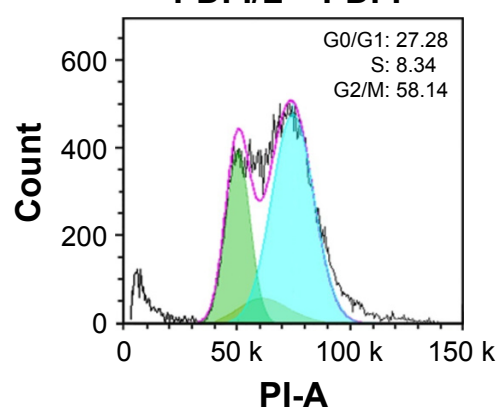

Figure 6 The in vitro synergistic effects of PDFI nanoparticles/laser irradiation combined with PDFP nanoparticles in MHCC-97H cells.

Notes: (A) The cytotoxicities of free PTX at different concentrations after treatments for $48 \mathrm{~h}$. (B) The cytotoxicities of PDFP nanoparticles combined with and without PDFI nanoparticles/laser irradiation (PDFI/L) at different PTX concentrations after treatments for $48 \mathrm{~h}$. The IR780 concentration was I $\mu \mathrm{g} / \mathrm{mL}$. The laser irradiation was carried out at $808 \mathrm{~nm}$ with a powder density of $2 \mathrm{~W} / \mathrm{cm}^{2}$ for $2 \mathrm{~min}$. $* p<0.05$ and $* * p<0.01$ for comparing these two treatment groups. (C) The apoptosis of cells at $48 \mathrm{~h}$ after various treatments (QI: dead cells; Q2: late apoptotic cells; Q3: early apoptotic cells; Q4: live cells). (D) The cell cycle distributions at $48 \mathrm{~h}$ after various treatments. The concentrations of IR780 and PTX were 1.5 and $5 \mu \mathrm{g} / \mathrm{mL}$, respectively, and all experiments were repeated at least 3 times.

Abbreviations: DOPE, I,2-dioleoyl-sn-glycero-3-phosphoethanolamine; PDFI, pullulan coated IR780-loaded DOPE/PF68 complex nanocore; PDFP, pullulan coated paclitaxel-loaded DOPE/PF68 complex nanocore; PF68, Pluronic F68; PTX, paclitaxel; FITC, fluorescein isothiocyanate.

in MHCC-97H cells. As shown in Figure S2, the laser irradiation itself hardly induced cell apoptosis, but remarkably enhanced the induction effect of DFI nanocores on cell apoptosis. The results of other treatment groups are shown in Figure 6C. PDFI nanoparticles significantly induced cell apoptosis after laser irradiation, which should be attributed to their potent phototherapeutic efficacy. Compared with free PTX, PDFP nanoparticles exhibited almost identical induction effect on cell apoptosis, indicating their strong cytotoxicty. However, the cells with combined treatment displayed a much higher apoptosis ratio at the same IR780 and PTX concentrations, further confirming the synergistic effects of phototherapy induced by PDFI nanoparticles and cytotoxic activity of PDFP nanoparticles. Meanwhile, we also evaluated the influence of PDFI nanoparticles/laser irradiation combined with PDFP nanoparticles on cell cycle in MHCC-97H cells. Cell cycle distributions after various treatments are shown in Figure 6D. PDFI nanoparticles either with or without laser irradiation did not induce significant cell cycle arrest. These results demonstrated that phototherapeutic effects of PDFI nanoparticles could not be caused by the cell cycle-dependent changes. Free PTX and PDFP nanoparticles both significantly induced the $\mathrm{G} 2 / \mathrm{M}$ cell cycle arrest. It was basically consistent with the results previously reported that PTX could bind and stabilize microtubules, cause cells to arrest at $\mathrm{G} 2 / \mathrm{M}$ phase in mitosis and result in cytotoxic responses. ${ }^{37}$ The cells with combined treatment were also arrested at G2/M phase, which should be mainly induced by chemotherapeutic effects of PDFP nanoparticles.

\section{In vivo distribution of PDFI nanoparticles}

To evaluate HCC-targeting and accumulating ability of nanoparticle system designed in this study, we assessed the biodistribution of PDFI nanoparticles in MHCC-97H tumor-bearing mice using the in vivo imaging technique. The fluorescence images of mice after intravenous injections of normal saline, free IR780, DFI nanocores and PDFI nanoparticles are shown in Figure 7A. Compared with free IR780, DFI nanocores and PDFI nanoparticles delayed the in vivo elimination of IR780 from the mouse body after administration. DFI nanocores were almost distributed in the whole body of mice during the first $8 \mathrm{~h}$, but PDFI nanoparticles began to enter the tumor at $4 \mathrm{~h}$ and reached a very high level of tumor accumulation at $8 \mathrm{~h}$. At $24 \mathrm{~h}$ after administration, 
A
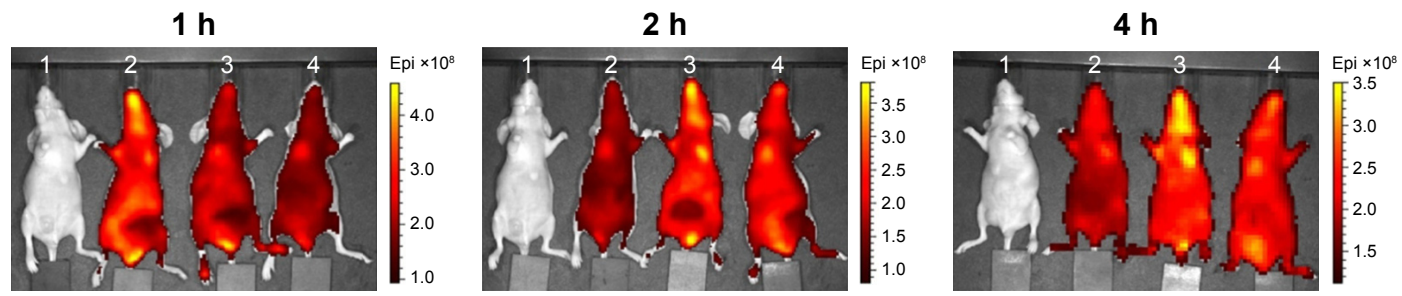

$8 \mathrm{~h}$

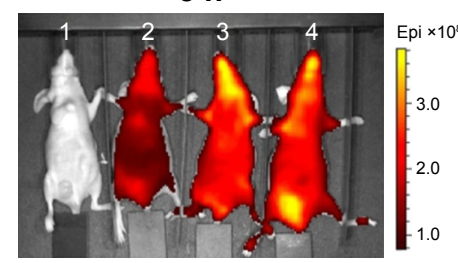

$24 \mathrm{~h}$

B
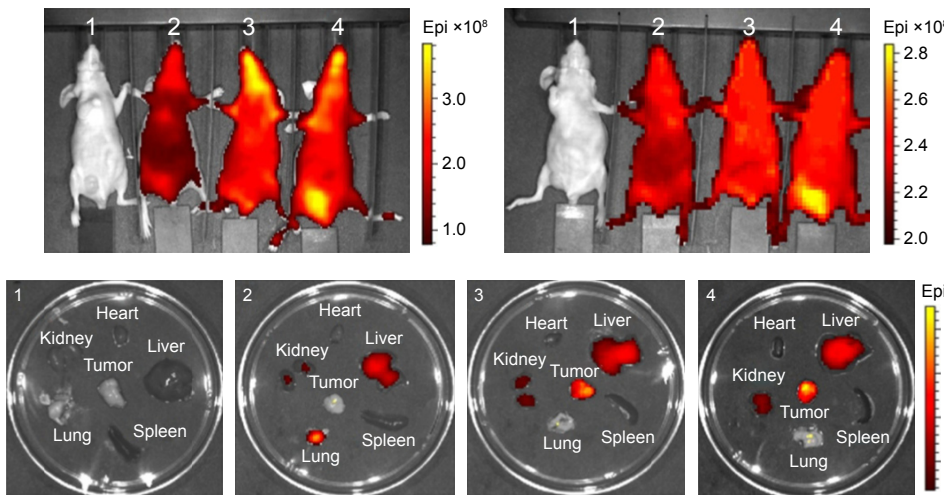

Figure 7 The hepatocellular carcinoma-targeting and accumulating capability of PDFI nanoparticles in MHCC-97H tumor-bearing mice.

Notes: (A) The fluorescence images of mice at different times after administrations through intravenous injection (I: the control mouse injected with normal saline; 2 , 3 and 4: the mice injected separately of free IR780, DFI nanocores and PDFI nanoparticles at the IR780 dose of $0.2 \mathrm{mg} / \mathrm{kg}$ ). (B) The fluorescence images of main organs and tumors removed from the mice at $24 \mathrm{~h}$ after administrations.

Abbreviations: DFI, IR780-loaded DOPE/PF68 complex; DOPE, I,2-dioleoyl-sn-glycero-3-phosphoethanolamine; PDFI, pullulan coated IR780-loaded DOPE/PF68 complex nanocore; PF68, Pluronic F68.

PDFI nanoparticles still exhibited an intensive fluorescence signal in the tumor, suggesting their excellent tumor-targeting and accumulating ability. Next, all mice were sacrificed and the tumors as well as major organs were collected for further imaging. The images are shown in Figure 7B. Free IR780 exhibited some residual distributions in the liver and lung, but DFI nanocores and PDFI nanoparticles were both mainly distributed in the tumor and liver. By contrast, PDFI nanoparticles evidently increased the tumor accumulation and slightly decreased the liver distribution of IR780, further confirming their tumor-targeting and accumulating ability. According to our previous reports, ${ }^{19,20}$ we believed that the EPR effect due to nanoscaled particle size and specific affinity of pullulan for ASGPR both played major roles in HCC-targeted delivery of PDFI nanoparticles in vivo.

\section{In vivo synergistic effects of combined treatment of PDFI nanoparticles/laser irradiation and PDFP nanoparticles}

We further evaluated the synergistic effects of combined treatment of PDFI nanoparticles/laser irradiation and PDFP nanoparticles in MHCC-97H tumor-bearing mice. The tumor growth curves after various treatments are shown in Figure 8A. Compared with the control, all treatments showed different inhibitory intensities on the tumor growth. PDFP nanoparticles had obviously higher anti-tumor efficacy than free PTX, which should be attributed to their HCC-targeted delivery. After laser irradiation, the tumor growth in mice treated with PDFI nanoparticles was almost completely suppressed, but began to recur on the eighth day with a rapid speed. It indicated that certain tumor cells survived PDFI nanoparticles-induced phototherapy. In contrast, the further treatment with PDFP nanoparticles significantly inhibited local tumor recurrence. Figure 8B shows the photos of mice after various treatments. The burn scars were clearly observed in mice at day 6 after treatment of PDFI nanoparticles/laser irradiation, and after that, gradually repaired with tumor recurrence. However, the tumor in mouse with further intravenous injection of PDFP nanoparticles was obviously smaller at 20 day. Besides, the tumors in combined treatment group had smallest sizes in all treatment groups (Figure 8C). All aforementioned results demonstrated that the strategy of combined treatment designed in this study could synergistically and efficiently inhibit the HCC growth in vivo.

Figure 8D shows the body weight changes of mice during various treatments. In the control group, the mouse body weights increased more or less with time over. However, free PTX caused the continuous decrease of body weights, indicating it had a certain degree of in vivo toxicity. By contrast, PDFP nanoparticles reversed this declining trend, meaning that HCC-targeted delivery of PDFP nanoparticles effectively 
A

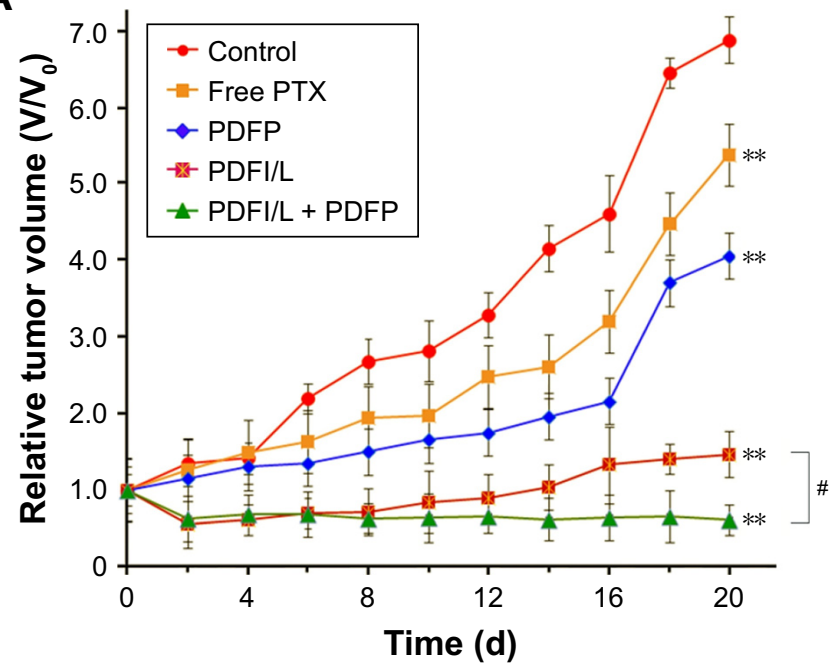

C

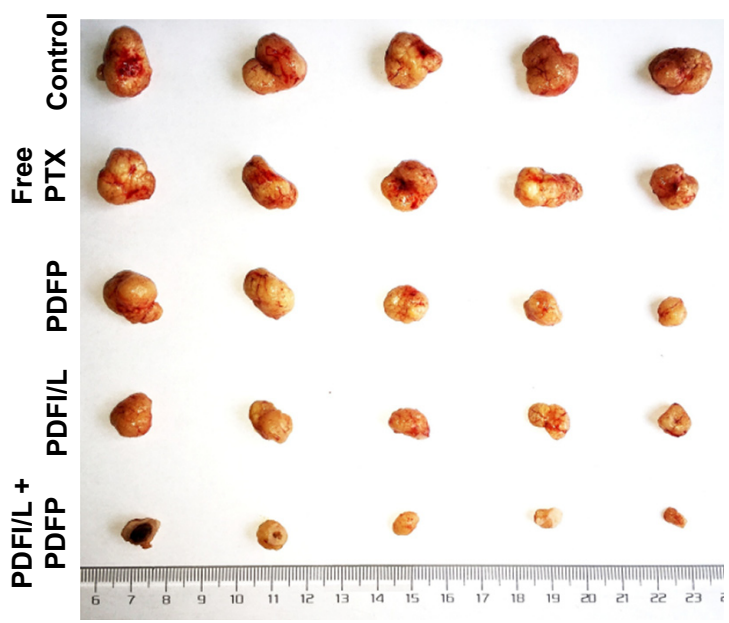

\begin{tabular}{l}
$E$ \\
\hline 0 \\
\hline 0
\end{tabular}
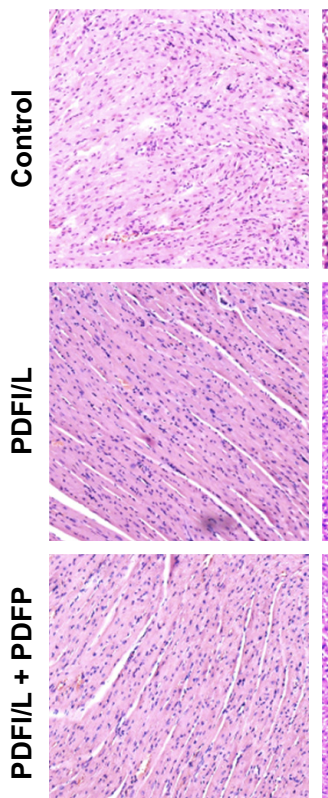
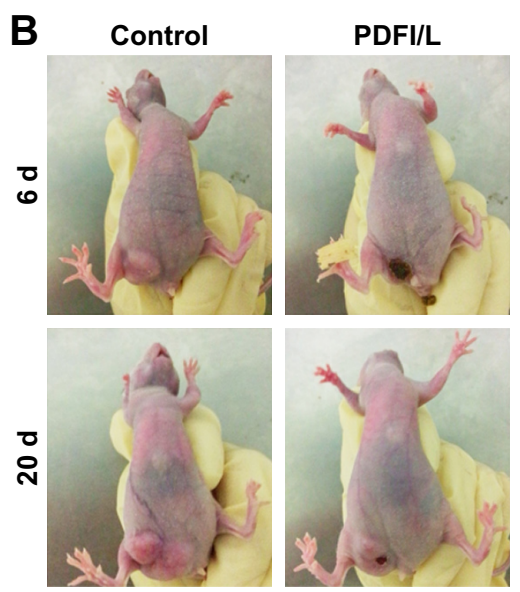

PDFI/L + PDFP
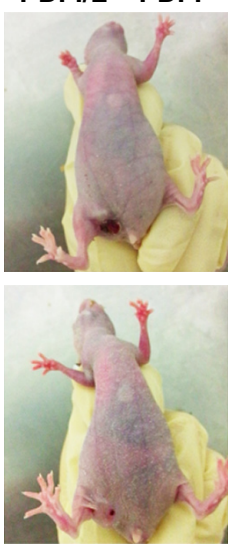

D

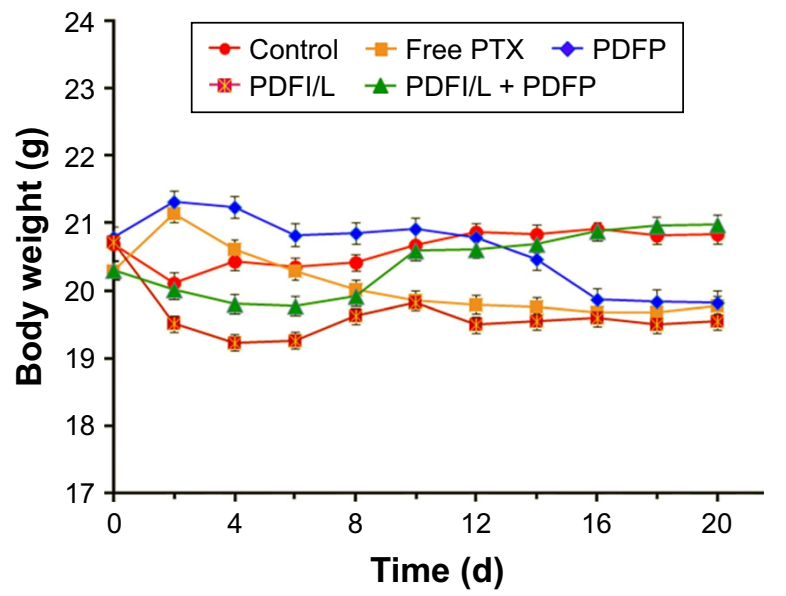

Lung
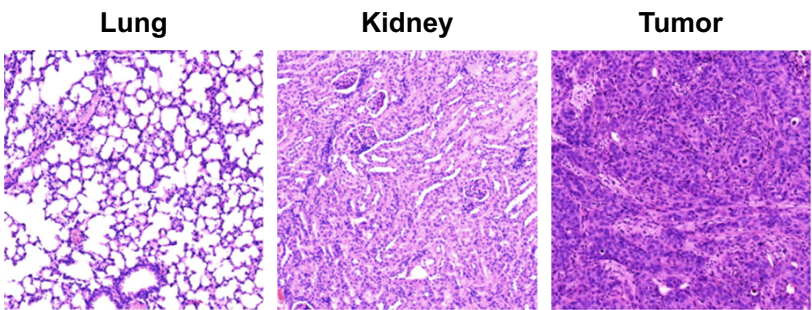

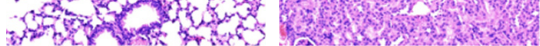
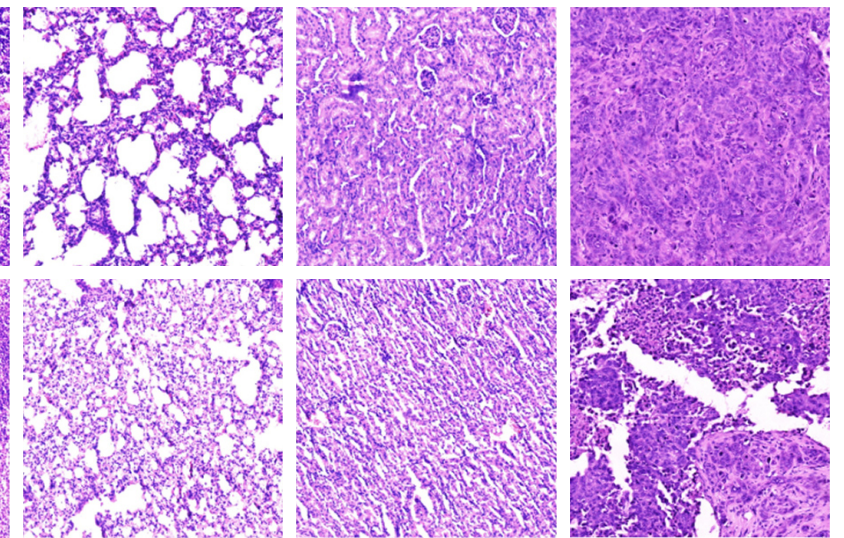


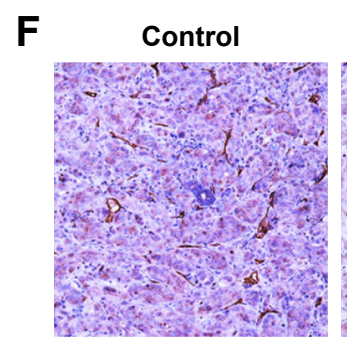

Free PTX

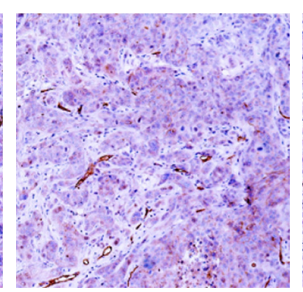

PDFP

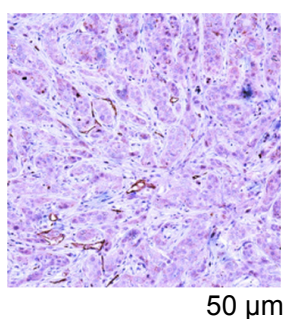

PDFI/L

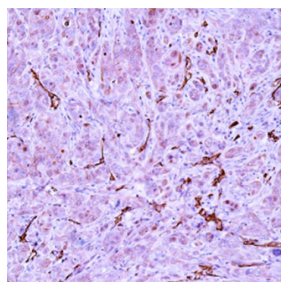

PDFI/L + PDFP

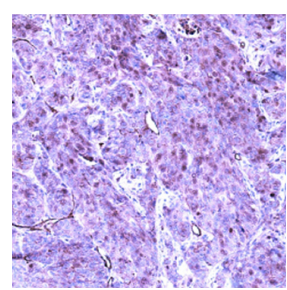

G

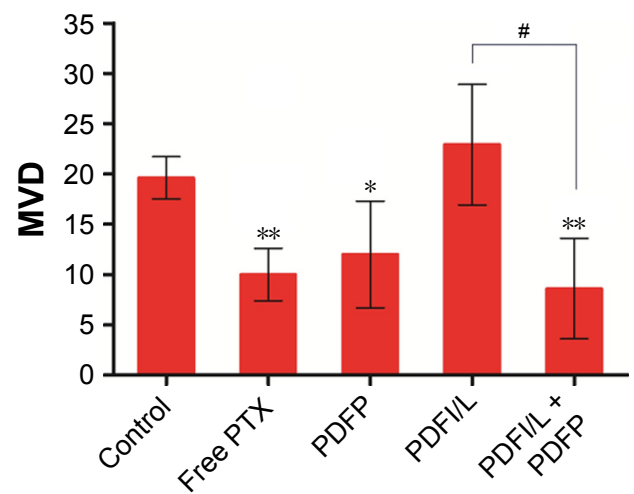

Figure 8 The in vivo synergistic effects of PDFI nanoparticles/laser irradiation combined with PDFP nanoparticles in MHCC-97H tumor-bearing mice.

Notes: (A) The growth curves of tumors in mice during the treatment period. PDFI nanoparticles were directly injected into the tumors at the IR780 dose of I00 $\mu g$ per mouse. The laser irradiation (L) was carried out locally on the tumor for $5 \mathrm{~min}$ at a power density of $2 \mathrm{~W} / \mathrm{cm}^{2}$. Free PTX and PDFP nanoparticles were injected into mice via the tail vein at the PTX dose of $5 \mathrm{mg} / \mathrm{kg}$. (B) The photos of mice at 6 and $20 \mathrm{~d}$ after various treatments. (C) The image of tumors removed from mice after various treatments. (D) The changes of mouse body weights during the treatment period. (E) The micrographs of H\&E-stained sections of main organs and tumors after various treatments. (F) The immunohistochemistry images of tumor sections stained with anti-CD3I antibody. Brown color represents positive staining of vascular epithelial cells. (G) The relative MVD of tumors. All results are mean $\pm S D(n=6) . * p<0.05$ and ${ }^{* *} p<0.01$ compared with the control; ${ }^{*} p<0.05$ for comparing two treatment groups.

Abbreviations: d, days; DOPE, I,2-dioleoyl-sn-glycero-3-phosphoethanolamine; MVD, microvessel densities; PDFI, pullulan coated IR780-loaded DOPE/PF68 complex nanocore; PDFP, pullulan coated paclitaxel-loaded DOPE/PF68 complex nanocore; PF68, Pluronic F68; PTX, paclitaxel.

alleviated in vivo toxicity of PTX. This toxicity-attenuation effect was also observed in other pullulan-based nanoparticle systems. ${ }^{20}$ The body weights of mice treated with PDFI nanoparticles/laser irradiation significantly decreased during the first 6 days, but afterwards increased slightly. It indicated that phototherapy induced by PDFI nanoparticles also had a little in vivo toxicity. However, by contrast, the mice receiving further treatment of PDFI nanoparticles maintained a relatively high level of body weights during the late treatment period, demonstrating the relatively low toxicity in vivo of the combined treatment strategy.

The micrographs of H\&E-stained sections of main organs and tumors are shown in Figure 8E. Almost no pathological changes and injuries were detectable in the main organs in mice after treatments of PDFI nanoparticles/laser irradiation combined with and without PDFP nanoparticles. However, compared with phototherapy triggered by PDFI nanoparticles alone, further treatment of PDFP nanoparticles caused more visible tumor necrosis and suppressed invasion of tumor cells, indicating significant synergistic effects of combined treatment. The micrographs of H\&E-stained organ and tumor sections in other treatment groups are shown in Figure S3. Obvious histopathological changes such as vascular congestion, tissue swelling and cell debris were visible in the liver of mice treated with free PTX, which was consistent with previously reported observation that PTX could induce the liver injury due to its distinct toxicity. ${ }^{20}$ However, no obvious abnormality was detected in the liver of mice treated with PDFP nanoparticles, demonstrating that the hepatotoxicity of PTX was remarkably reduced by HCC-targeted delivery of PDFP nanoparticles. In view of the strong antiangiogensis activity of PTX, ${ }^{38}$ we further evaluated the intratumoral vascularization after various treatments using the immunohistochemical staining of CD31. The results are shown in Figure 8F and G. Compared with the control, the tumor angiogenesis was slightly accelerated in mice receiving treatment of PDFI nanoparticles/laser irradiation. We believed it was perhaps because the inflammation after PTT stimulated tumor angiogenesis. All other treatments exhibited significant antiangiogenesis activities. More interestingly, further treatment of PDFP nanoparticles effectively prevented tumor angiogenesis induced by PDFI nanoparticles/irradiation. Considering important roles of angiogenesis in the local growth and metastasis of a variety of malignant tumors, ${ }^{38}$ we deduced that this combined treatment strategy would be favorable for inhibiting the metastasis of HCC.

\section{Conclusion}

In this study, a simple but effective nanoparticle system was designed for carrying IR780 and PTX, thus hoping to effectively combine PTT/PDT and chemotherapy to treat HCC. At the cellular level, PDFI nanoparticles/laser irradiation combined with PDFP nanoparticles significantly inhibited the cell growth and induced the cell apoptosis and 
cell cycle arrest at G2/M phase. At the animal level, the combined treatment effectively inhibited the tumor growth and angiogenesis in HCC tumor-bearing mice. Our results suggested that PDF nanoparticle system can efficiently combine PTT/PDT and chemotherapy to treat HCC. However, molecular mechanisms of synergistic effects such as cell apoptosis induction, antiangiogenesis and HCC-targeted delivery should be further illuminated for the clinical application of this novel combined strategy.

\section{Acknowledgments}

This work was supported by the National Natural Science Foundation of China (grant numbers 81371671, 81573005 and 81501575) and the Tianjin 13th Five-year and TMU Talent Project (grant number 2016KJ0308).

\section{Disclosure}

The authors report no conflicts of interest in this work.

\section{References}

1. Chan HL, Hui AY, Wong ML, et al. Genotype C hepatitis B virus infection is associated with an increased risk of hepatocellular carcinoma. Gut. 2004;53(10):1494-1498.

2. Harnois DM. Hepatitis $C$ virus infection and the rising incidence of hepatocellular carcinoma. Mayo Clin Proc. 2012;87(1):7-8.

3. Wang JH, Wang CC, Hung CH, et al. Survival comparison between surgical resection and radiofrequency ablation for patients in BCLC very early/early stage hepatocellular carcinoma. J Hepatol. 2012;56(2): 412-418.

4. Bruix J, Reig M, Sherman M. Evidence-based diagnosis, staging, and treatment of patients with hepatocellular carcinoma. Gastroenterology. 2016;150(4):835-853.

5. Guha C, Kavanagh BD. Hepatic radiation toxicity: avoidance and amelioration. Semin Radiat Oncol. 2011;21(4):256-263.

6. Yan J, Zhou Y, Chen D, et al. Effects of mitochondrial translocation of telomerase on drug resistance in hepatocellular carcinoma cells. J Cancer. 2015;6(2):151-159.

7. Wu P-C, Shieh D-B, Cheng F-Y. Nanomaterial-mediated photothermal cancer treatment: the pivotal role of cellular uptake on photothermal therapeutic efficacy. RSC Adv. 2014;4(95):53297-53306.

8. O'Neal DP, Hirsch LR, Halas NJ, et al. Photo-thermal tumor ablation in mice using near infrared-absorbing nanoparticles. Cancer Lett. 2004; 209(2):171-176.

9. Chen Q, Liang C, Wang X, et al. An albumin-based theranostic nanoagent for dual-modal imaging guided photothermal therapy to inhibit lymphatic metastasis of cancer post surgery. Biomaterials. 2014;35(34): 9355-9362.

10. Zou L, Wang H, He B, et al. Current approaches of photothermal therapy in treating cancer metastasis with nanotherapeutics. Theranostics. 2016;6(6):762-772.

11. Zeng Y, Zhang D, Wu M, et al. Lipid-AuNPs@PDA nanohybrid for $\mathrm{MRI} / \mathrm{CT}$ imaging and photothermal therapy of hepatocellular carcinoma. ACS Appl Mater Interfaces. 2014;6(16):14266-14277.

12. Turro NJ. The role of intersystem crossing steps in singlet oxygen chemistry and photo-oxidations. Tetrahedron. 1985;41(11):2089-2098.

13. Barra F, Roscetto E, Soriano AA, et al. Photodynamic and antibiotic therapy in combination to fight biofilms and resistant surface bacterial infections. Int J Mol Sci. 2015;16(9):20417-20430.
14. Sharma P, Bengtsson NE, Walter GA, et al. Gadolinium-doped silica nanoparticles encapsulating indocyanine green for near infrared and magnetic resonance imaging. Small. 2012;8(18):2856-2868.

15. Wang K, Zhang Y, Wang J, et al. Self-assembled IR780-loaded transferrin nanoparticles as an imaging, targeting and PDT/PTT agent for cancer therapy. Sci Rep. 2016;6:27421.

16. Guo F, Yu M, Wang J, et al. The mitochondria-targeted and IR780regulated theranosomes for imaging and enhanced photodynamic/ photothermal therapy. RSC Adv. 2016;6(14):11070-11076.

17. Yan F, Duan W, Li Y, et al. NIR-laser-controlled drug release from DOX/IR-780-loaded temperature-sensitive-liposomes for chemophotothermal synergistic tumor therapy. Theranostics. 2016;6(13): 2337-2351.

18. Kaneo Y, Tanaka T, Nakano T, Yamaguchi Y. Evidence for receptormediated hepatic uptake of pullulan in rats. $J$ Control Release. 2001; 70(3):365-373.

19. Liu Y, Wang Y, Zhang C, et al. Core-shell nanoparticles based on pullulan and poly(beta-amino) ester for hepatoma-targeted codelivery of gene and chemotherapy agent. ACS Appl Mater Interfaces. 2014; 6(21):18712-18720.

20. Zhang C, An T, Wang D, et al. Stepwise pH-responsive nanoparticles containing charge-reversible pullulan-based shells and poly(betaamino ester)/poly(lactic-co-glycolic acid) cores as carriers of anticancer drugs for combination therapy on hepatocellular carcinoma. J Control Release. 2016;226:193-204.

21. Elzainy A, Gu X, Estelle F, et al. Hydroxyzine- and cetirizine-loaded liposomes: effect of duration of thin film hydration, freeze-thawing, and changing buffer $\mathrm{pH}$ on encapsulation and stability. Drug Dev Ind Pharm. 2005;31(3):281-291.

22. Aditya NP, Patankar S, Madhusudhan B, et al. Arthemeter-loaded lipid nanoparticles produced by modified thin-film hydration: pharmacokinetics, toxicological and in vivo anti-malarial activity. Eur $J$ Pharm Sci. 2010;40(5):448-455.

23. Liu Y, Wan G, Guo H, et al. A multifunctional nanoparticle system combines sonodynamic therapy and chemotherapy to treat hepatocellular carcinoma. Nano Res. 2017;10(3):834-855.

24. Li J, Wang X, Zhang T, et al. A review on phospholipids and their main applications in drug delivery systems. Asian J Pharm Sci. 2015;10(2): 81-98.

25. Shin J. Acid-triggered release via dePEGylation of DOPE liposomes containing acid-labile vinyl ether PEG-lipids. J Control Release. 2003; 91(1):187-200.

26. Singh RS, Kaur N, Rana V, Kennedy JF. Pullulan: a novel molecule for biomedical applications. Carbohydr Polym. 2017;171:102-121.

27. D'Souza AA, Devarajan PV. Asialoglycoprotein receptor mediated hepatocyte targeting-strategies and applications. J Control Release. 2015;203:126-139.

28. Huh KM, Lee SC, Cho YW, et al. Hydrotropic polymer micelle system for delivery of paclitaxel. J Control Release. 2005;101(1):59-68.

29. Surapaneni MS, Das SK, Das NG. Designing paclitaxel drug delivery systems aimed at improved patient outcomes: current status and challenges. ISRN Pharmacol. 2012;2012(4):623139.

30. Lin H, Shen Y, Chen D, et al. Feasibility study on quantitative measurements of singlet oxygen generation using singlet oxygen sensor green. J Fluoresc. 2012;23(1):41-47.

31. Price M, Reiners JJ, Santiago AM, Kessel D. Monitoring singlet oxygen and hydroxyl radical formation with fluorescent probes during photodynamic therapy. Photochem Photobiol. 2009;85(5):1177-1181.

32. Flors $\mathrm{C}$, Fryer MJ, Waring $\mathrm{J}$, et al. Imaging the production of singlet oxygen in vivo using a new fluorescent sensor, singlet oxygen sensor green. J Exp Bot. 2006;57(8):725-734.

33. Kim HW, Cho SJ, Kim BY, et al. Pogostemon cablin as ROS scavenger in oxidant-induced cell death of human neuroglioma cells. Evid Based Complement Alternat Med. 2010;7(2):239-247. 
34. Li W, Peng J, Tan L, et al. Mild photothermal therapy/photodynamic therapy/chemotherapy of breast cancer by Lyp-1 modified docetaxel/ IR820 co-loaded micelles. Biomaterials. 2016;106:119-133.

35. Zhang $\mathrm{P}, \mathrm{Hu} \mathrm{C}$, Ran $\mathrm{W}$, et al. Recent progress in light-triggered nanotheranostics for cancer treatment. Theranostics. 2016;6(7):948-968.

36. Lv R, Yang P, He F, et al. An imaging-guided platform for synergistic photodynamic/photothermal/chemo-therapy with $\mathrm{pH} /$ temperatureresponsive drug release. Biomaterials. 2015;63:115-127.
37. Shu CH, Yang WK, Shih YL, et al. Cell cycle G2/M arrest and activation of cyclin-dependent kinases associated with low-dose paclitaxelinduced sub-G1 apoptosis. Apoptosis. 1997;2(5):463-470.

38. Bocci G, Di Paolo A, Danesi R. The pharmacological bases of the antiangiogenic activity of paclitaxel. Angiogenesis. 2013;16(3): $481-492$. 


\section{Supplementary materials}

Table SI The size and zeta potential changes of PDF nanoparticles during storage in $\mathrm{pH} 7.4$ PBS solution

\begin{tabular}{llll}
\hline Storage time (days) & Size $(\mathbf{n m})$ & PDI & Zeta potential (mV) \\
\hline 1 & 226.9 & 0.226 & -4.91 \\
2 & 224.9 & 0.246 & -5.80 \\
3 & 209.4 & 0.275 & -6.14 \\
4 & 222.1 & 0.301 & -6.56 \\
\hline
\end{tabular}

Note: Sizes, PDIs and zeta potentials of PDF nanoparticles are presented as the average values from 3 independent experiments.

Abbreviations: DOPE, I,2-dioleoyl-sn-glycero-3-phosphoethanolamine; PBS, phosphate buffer saline; PDF, pullulan coated DOPE/PF68 complex nanocore; PDI, polydispersity index; PF68, Pluronic F68.

Table S2 The characteristic parameters of DFI nanoparticles with different weight ratio of IR780 and DOPE/PF68

\begin{tabular}{llllll}
\hline IR780/DOPE/PF68 (w/w/w) & Size $(\mathbf{n m})$ & PDI & Zeta potential $(\mathbf{m V})$ & LC $(\%)$ & EE $(\%)$ \\
\hline I/I0/50 & 314.5 & 0.326 & 3.6 & 9.5 & 3.9 \\
$2.5 / 10 / 50$ & 298.0 & 0.276 & 4.8 & 8.0 \\
$5 / 10 / 50$ & 178.6 & 0.125 & 11.9 & 93.6 \\
$10 / 10 / 50$ & 172.4 & 0.381 & 6.5 & 10.8 & 96.0 \\
\hline
\end{tabular}

Note: Sizes, PDIs, zeta potentials, IR780-LCs and EEs of DFI nanoparticles are presented as the average values from 3 independent experiments.

Abbreviations: DFI, IR780-loaded DOPE/PF68 complex; DOPE, I,2-dioleoyl-sn-glycero-3-phosphoethanolamine; EE, encapsulation efficiency; LC, loading content; PDI, polydispersity index; PF68, Pluronic F68; w/w/w, weight ratio.

Table S3 The drug-loading parameters of DFI, PDFI, DFP and PDFP nanoparticles prepared under the optimal conditions

\begin{tabular}{lllll}
\hline Drug formulation & DFI & PDFI & DFP & \\
\hline LC (\%) & 8.0 & 4.6 & 7.7 & 3.8 \\
EE (\%) & 96.0 & 92.0 & 92.8 & 76.0 \\
\hline
\end{tabular}

Note: The LCs and EEs of IR780 and paclitaxel were determined, respectively, by the ultraviolet-visible and ultraperformance liquid chromatography methods.

Abbreviations: DFI, IR780-loaded DOPE/PF68 complex; DFP, paclitaxel-loaded DOPE/PF68 complex; EE, encapsulation efficiency; LC, loading content; PDFI, pullulan coated IR780-loaded DOPE/PF68 complex nanocore; PDFP, pullulan coated paclitaxel-loaded DOPE/PF68 complex nanocore.
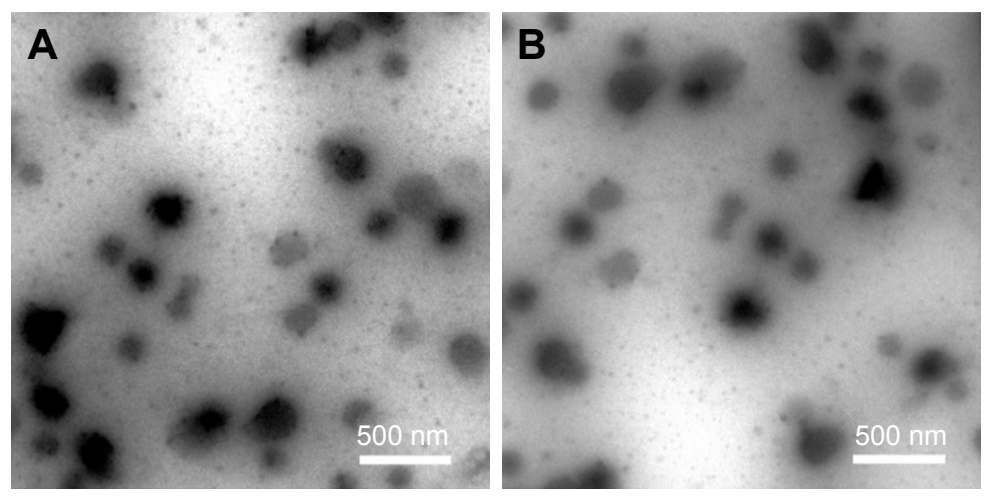

Figure SI The transmission electron microscope images of PDFI (A) and PDFP nanoparticles (B) in deionized water.

Note: The nanoparticle concentrations were about $0.5 \mathrm{mg} / \mathrm{mL}$.

Abbreviations: DOPE, I,2-dioleoyl-sn-glycero-3-phosphoethanolamine; PDFI, pullulan coated IR780-loaded DOPE/PF68 complex nanocore; PDFP, pullulan coated paclitaxel-loaded DOPE/PF68 complex nanocore; PF68, Pluronic F68. 

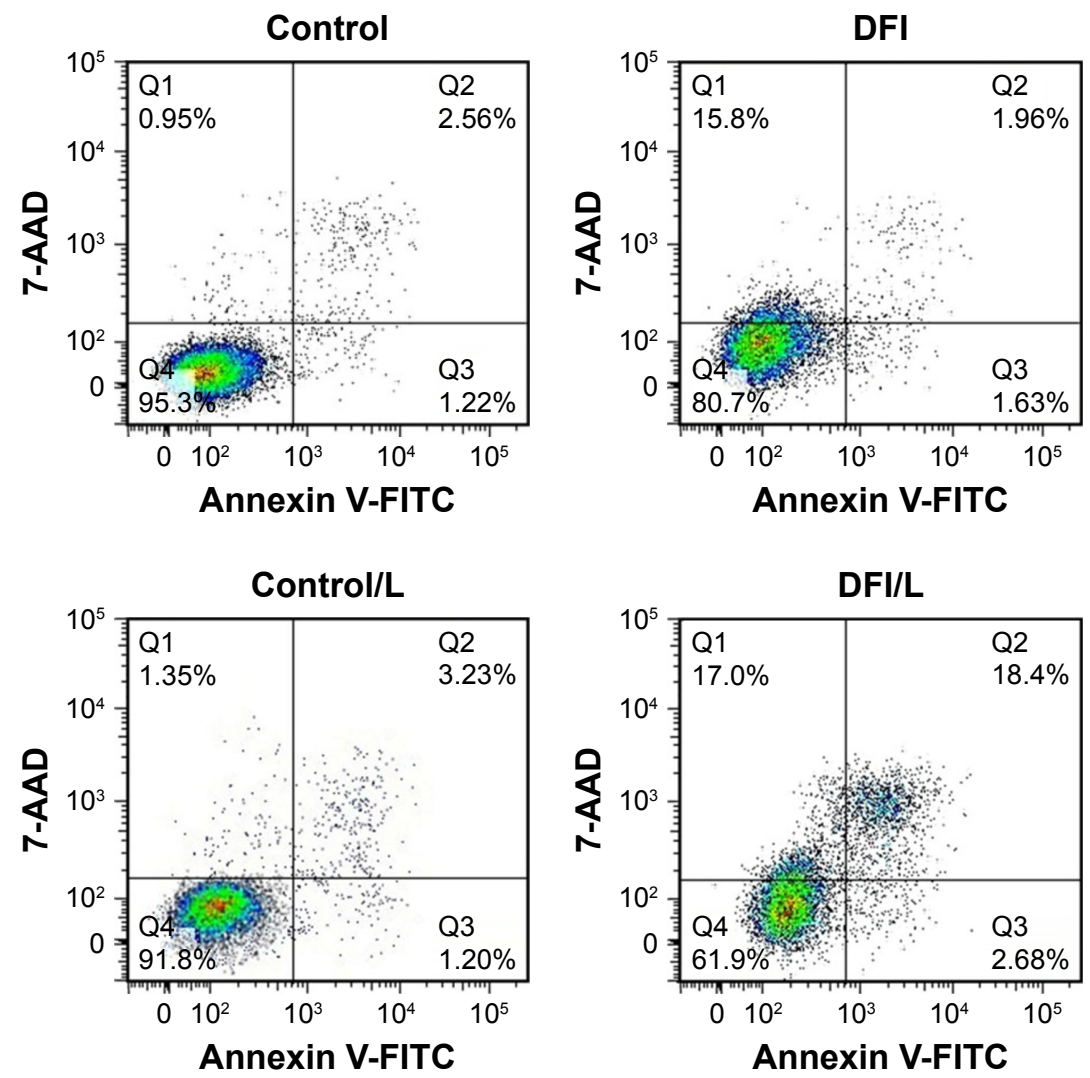

Figure S2 The apoptosis of MHCC-97H cells at $48 \mathrm{~h}$ after treatments of DFI nanocores combined with and without NIR laser irradiation (808 nm, 2 min). Notes: Q1: dead cells; Q2: late apoptotic cells; Q3: early apoptotic cells; Q4: live cells. The concentrations of IR780 were I.5 $\mu \mathrm{g} / \mathrm{mL}$ and all experiments were repeated at least 3 times.

Abbreviations: DFI, IR780-loaded DOPE/PF68 complex; DOPE, I,2-dioleoyl-sn-glycero-3-phosphoethanolamine; NIR, near-infrared; FITC, fluorescein isothiocyanate; PF68, Pluronic F68.
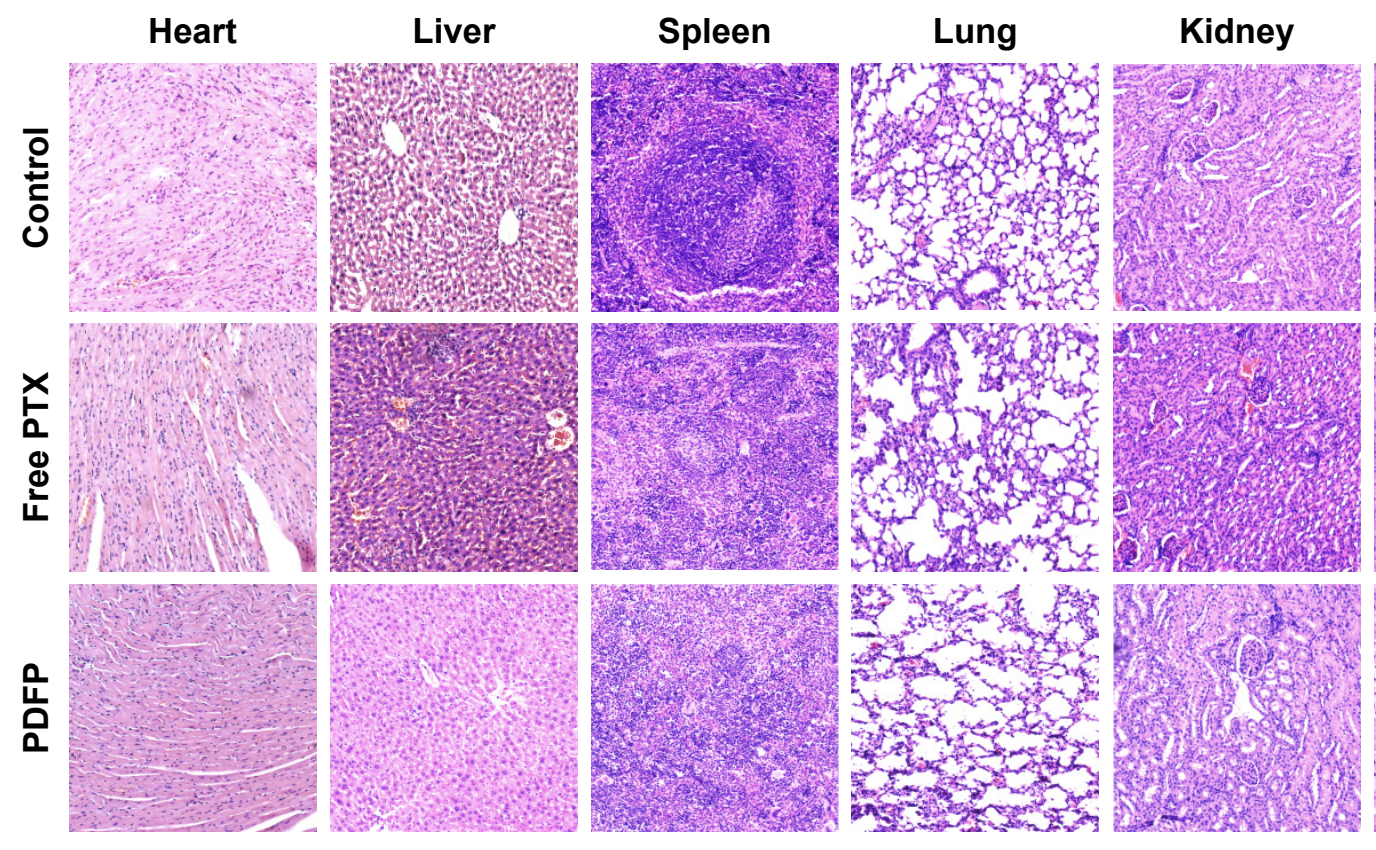

Tumor
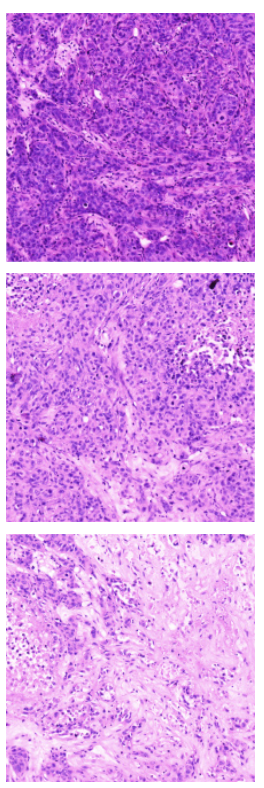

$200 \mu \mathrm{m}$

Figure S3 The micrographs of H\&E stained sections of main organs and tumors removed from the mice after treatments of normal saline (the control), free PTX and PDFP nanoparticles at the PTX dose was $5 \mathrm{mg} / \mathrm{kg}$.

Note: The mice were administrated via intravenous injection every other day for consecutive 3 times.

Abbreviations: DOPE, I,2-dioleoyl-sn-glycero-3-phosphoethanolamine; H\&E, hematoxylin and eosin; PDFP, pullulan coated paclitaxel-loaded DOPE/PF68 complex nanocore; PF68, Pluronic F68; PTX, paclitaxel. 


\section{Publish your work in this journal}

The International Journal of Nanomedicine is an international, peerreviewed journal focusing on the application of nanotechnology in diagnostics, therapeutics, and drug delivery systems throughout the biomedical field. This journal is indexed on PubMed Central, MedLine, CAS, SciSearch $\AA$, Current Contents $\AA /$ Clinical Medicine,

Journal Citation Reports/Science Edition, EMBase, Scopus and the Elsevier Bibliographic databases. The manuscript management system is completely online and includes a very quick and fair peer-review system, which is all easy to use. Visit http://www.dovepress.com/ testimonials.php to read real quotes from published authors.

Submit your manuscript here: http://www.dovepress.com/international-journal-of-nanomedicine-journal 